- Supporting Information -

\title{
Dipnictogen f-Element Chemistry: A Diphosphorus Uranium Complex
}

Jingzhen Du, ${ }^{a}$ David Hunger, ${ }^{\mathrm{b}}$ John A. Seed, ${ }^{\mathrm{a}}$ Jonathan D. Cryer, ${ }^{\mathrm{a}}$ David M. King, ${ }^{\mathrm{c}}$

Ashley J. Wooles, ${ }^{a}$ Joris van Slageren, ${ }^{\mathrm{b}}$ and Stephen T. Liddle ${ }^{\mathrm{a}, *}$

${ }^{a}$ Department of Chemistry, The University of Manchester, Oxford Road, Manchester, M13 9PL, UK.

${ }^{\mathrm{b}}$ Institute of Physical Chemistry, University of Stuttgart, Pfaffenwaldring 55, D-70569 Stuttgart, Germany.

c School of Chemistry, University of Nottingham, University Park, Nottingham, NG7 2RD, UK.

*e-mail: steve.liddle@manchester.ac.uk

\section{Table of Contents}

Experimental Details

Molecular Structures

Spectroscopic and Magnetic Data

Theoretical Calculations

References S34 


\section{Experimental Details}

\section{General}

All manipulations were carried out under an inert atmosphere of dry dinitrogen using Schlenk techniques, or an MBraun UniLab glovebox operating under an atmosphere of dry dinitrogen. $\mathrm{DCM}, \mathrm{Et}_{2} \mathrm{O}$ and pentane solvents were dried by passage through activated alumina towers and degassed before use. Benzene was distilled from potassium. All solvents were stored over potassium mirrors except for ethers which were stored over activated $4 \AA$ sieves. Deuterated solvents were distilled from potassium, degassed by three freeze-pump-thaw cycles, and stored under dinitrogen prior to use. [(Tren $\left.\left.{ }^{\mathrm{TIPS}}\right) \mathrm{U}^{\mathrm{III}}\right](\mathbf{1}),{ }^{1}$ anthracene-P-H (2)/$\mathrm{NMe}_{2}(4) /-\mathrm{Bu}^{\mathrm{t} /} /\left(\mathrm{BPh}_{3}\right) \mathrm{Na}\left(\mathrm{OEt}_{2}\right)_{2} /-\mathrm{P}$-anthracene reagents were prepared using literature methods. $^{2-5}$ Single crystals were examined variously on either a) an Oxford Diffraction SuperNova Atlas CCD diffractometer using mirror-monochromated MoKa radiation $(\lambda=$ $0.71073 \AA$ ), b) a Rigaku XtalLAB Synergy-S diffractometer equipped with a Hypix $6000 \mathrm{HE}$ photon counting pixel array detector with mirror-monochromated $\mathrm{Mo}$ or $\mathrm{Cu} \mathrm{K} \alpha$ radiation $(\lambda=$ 0.71073 or $1.5418 \AA$ ), or c) a Rigaku FR-X diffractometer equipped with a Hypix $6000 \mathrm{HE}$ photon counting pixel array detector with mirror-monochromated $\operatorname{MoK} \alpha(\lambda=0.71073 \AA)$ or $\mathrm{CuK} \alpha(\lambda=1.5418 \AA)$ radiation. Intensities were integrated from a sphere of data recorded on narrow $\left(1.0^{\circ}\right)$ frames by $\omega$ rotation. Cell parameters were refined from the observed positions of all strong reflections in each data set. Gaussian grid face-indexed absorption corrections with a beam profile correction were applied. The structures were solved either by dual methods using SHELXT $^{6}$ and all non-hydrogen atoms were refined by full-matrix leastsquares on all unique F2 values with anisotropic displacement parameters with exceptions noted in the respective cif files. Hydrogen atoms were refined with constrained geometries and riding thermal parameters; Uiso(H) was set at 1.2 (1.5 for methyl groups) times Ueq of the parent atom. The largest features in final difference syntheses were close to heavy atoms 
and were of no chemical significance. CrysAlisPro was used for control and integration, ${ }^{7}$ and SHELXL and Olex2 were employed for structure refinement. ${ }^{8,9}$ ORTEP-3 and POV-Ray were employed for molecular graphics. ${ }^{10,11}{ }^{1} \mathrm{H}$ and ${ }^{29} \mathrm{Si}\left\{{ }^{1} \mathrm{H}\right\}$ spectra were recorded on a Bruker 400 spectrometer operating at 400 and $79 \mathrm{MHz}$, respectively; chemical shifts are quoted in ppm and are relative to TMS $\left({ }^{1} \mathrm{H},{ }^{29} \mathrm{Si}\right)$. ATR-IR spectra were recorded on a Bruker Alpha spectrometer with a Platinum-ATR module in the glovebox. Raman spectra were recorded on a Horiba XploRA Plus Raman microscope with a $638 \mathrm{~nm}$ laser with a power of $1.5 \mathrm{~mW}$. The power was adjusted using a power filter for each complex to inhibit sample decomposition. UV/Vis/NIR spectra were recorded on a Perkin Elmer LMDTM 750 spectrometer. Data were collected in a $1 \mathrm{~mm}$ path-length cuvette loaded in an MBraun glovebox and were run versus the appropriate solvent. Static variable-temperature magnetic moment data were recorded in applied dc fields of $0.1(1.8-50 \mathrm{~K})$ and $1(40-300 \mathrm{~K})$ Tesla on a Quantum Design MPMS3 superconducting quantum interference device (SQUID) magnetometer using recrystallized powdered samples. Care was taken to ensure complete thermalization of the sample before each data point was measured and samples were immobilized in an eicosane matrix to prevent sample reorientation during measurements. Diamagnetic corrections were applied for using tabulated Pascal constants and measurements were corrected for the effect of the blank sample holders (flame sealed Wilmad NMR tube and straw) and eicosane matrix. CHN microanalyses were carried out by Martin Jennings and Anne Davies at the University of Manchester.

\section{Synthesis and Isolation of $\left[\left\{\left(\text { Tren }^{T I P S}\right) U^{I V}\right\}_{2}(\mu-P H)\right](3)$}

A colorless solution of $2(0.11 \mathrm{~g}, 0.5 \mathrm{mmol})$ in benzene $(2 \mathrm{~mL})$ was added to a dark purple solution of $1(0.85 \mathrm{~g}, 1 \mathrm{mmol})$ in benzene $(4 \mathrm{~mL})$ in a small glass vial, and the mixture immediately turned into a dark red solution. The reaction was left without stirring for 16 
hours to afford dark red crystals of $\left[\left\{\left(\operatorname{Tren}^{\mathrm{TIPS}}\right) \mathrm{U}^{\mathrm{IV}}\right\}_{2}(\mu-\mathrm{PH})\right](\mathbf{3}),{ }^{12}$ which was isolated as crystalline solid by decanting the mother liquor, washing with pentane $(2 \times 1 \mathrm{~mL})$, and then drying in vacuo. Yield: $0.54 \mathrm{~g}, 62 \%$ (by U content). All characterization data matched those of an authentic sample. ${ }^{12}$ Using excess $\mathbf{2}$ also only produces $\mathbf{3}$ with the excess $\mathbf{2}$ decomposed to anthracene.

\section{Synthesis and Isolation of $\left.\left[\left(\operatorname{Tren}^{T I P S}\right) U^{I V}\right\}_{2}\left(\mu-\eta^{2}: \eta^{2}-P_{2}\right)\right](5)$}

A pale-yellow solution of $4(0.13 \mathrm{~g}, 0.5 \mathrm{mmol})$ in benzene $(2 \mathrm{~mL})$ was added to a dark purple solution of $1(0.85 \mathrm{~g}, 1.0 \mathrm{mmol})$ in benzene $(4 \mathrm{~mL})$. The mixture slowly turned dark red after 24 hours without stirring, precipitating dark red crystals of $\left[\left\{\left(\operatorname{Tren}^{\mathrm{TIPS}}\right) \mathrm{U}^{\mathrm{IV}}\right\}_{2}\left(\mu-\eta^{2}: \eta^{2}-\mathrm{P}_{2}\right)\right](\mathbf{5})$. The crystalline solid was isolated by decanting the mother liquor, washing with benzene $(2 \times$ $1 \mathrm{~mL}$ ), pentane $(2 \times 1 \mathrm{~mL})$, and then drying in vacuo. Yield: $0.40 \mathrm{~g}, 45 \%$ (by U content, maximum $50 \%$ yield). Using excess 4 also only produces 5 with the excess 4 left unreacted. In addition, 1 does not react with Anthracene- $\mathrm{P}\left(\mathrm{BPh}_{3}\right) \mathrm{Na}\left(\mathrm{OEt}_{2}\right)_{2}$ or Anthracene- $\mathrm{PBu}$, reflecting the more reactive nature of Anthracene-PH/ $\mathrm{NMe}_{2}$ as phosphinidene group transfer reagents. Anal. Calcd for $\mathrm{C}_{66} \mathrm{H}_{150} \mathrm{~N}_{8} \mathrm{Si}_{6} \mathrm{P}_{2} \mathrm{U}_{2}: \mathrm{C}, 44.98 ; \mathrm{H}, 8.58 ; \mathrm{N}, 6.36 \%$. Found: $\mathrm{C}, 45.49 ; \mathrm{H}$, 8.78; N, 6.22\%. ATR-IR v/cm-1: 2942 (br, w), 2890 (br, m), 2826 (m), 1459 (m), 1381 (w), 1360 (w), 1334 (w), 1271 (w), 1249 (w), $1140(\mathrm{w}), 1062$ (m), 1031 (m), 1008 (w), 931 (s), 877 (s), 794 (s), 722 (vs), 671 (s), 628 (m), 571 (w), 543 (w), 517 (w), 444 (w). Raman v/cm' $2930(\mathrm{~m}), 2866(\mathrm{~s}), 1466(\mathrm{w}), 1235(\mathrm{w}), 1067(\mathrm{w}), 962(\mathrm{w}), 880(\mathrm{~m}), 808(\mathrm{w}), 590(\mathrm{~m}), 540$ (br, w), 462 (br, w). Solution NMR and UV/Vis/NIR spectra and solution magnetic moment (Evan's method) could not be obtained as dimeric $\mathbf{5}$ is insoluble in common solvents such benzene, toluene and THF. 


\section{Synthesis and Isolation of [(Tren $\left.\left.{ }^{T I P S}\right) U^{I V}\left(\mathrm{NMe}_{2}\right)\right](6)$}

From the reaction that produced $\mathbf{5}$, removal of volatiles from the combined mother liquor and the washings gave a brown solid residue. Pentane $(4 \mathrm{~mL})$ was added to the residue to afford a brown solution and slurry, which was stored at $-30{ }^{\circ} \mathrm{C}$ for 4 hours to ensure all anthracene compounds precipitated. After filtration, the brown solution was concentrated to $\sim 2 \mathrm{~mL}$ and stored at $-30{ }^{\circ} \mathrm{C}$ for 24 hours, giving $\left[\left(\operatorname{Tren}^{\mathrm{TIPS}}\right) \mathrm{U}^{\mathrm{IV}}\left(\mathrm{NMe}_{2}\right)\right](6)$ as dark brown crystals. The crystalline solid was isolated by decanting the mother liquor, washing with cold pentane (1 $\mathrm{mL}$ ), and then drying in vacuo. Yield: $0.30 \mathrm{~g}, 34 \%$ (by U content, maximum $50 \%$ yield). Anal. Calcd for $\mathrm{C}_{35} \mathrm{H}_{81} \mathrm{~N}_{5} \mathrm{Si}_{3} \mathrm{U}$ : C, 47.00; H, 9.13; N, 7.83\%. Found: C, 46.84; H, 9.32; N, 7.54\%. ${ }^{1} \mathrm{H}$ NMR (400 MHz, $\left.\mathrm{C}_{6} \mathrm{D}_{6}, 298 \mathrm{~K}\right): \delta-12.48$ (br, 54H, $\left.\mathrm{CH}\left(\mathrm{CH}_{3}\right)_{2}\right),-11.82$ (br, 9H, $\left.\mathrm{CH}\left(\mathrm{CH}_{3}\right)_{2}\right), 4.87$ (br, 6H, N(CH3) $\left.)_{2}\right), 60.00$ (br, 6H, $\left.\mathrm{CH}_{2} \mathrm{CH}_{2}\right), 92.90$ (br, 6H, $\mathrm{CH}_{2} \mathrm{CH}_{2}$ ). ${ }^{29} \mathrm{Si}\left\{{ }^{1} \mathrm{H}\right\}$ NMR (79 MHz, $\left.\mathrm{C}_{6} \mathrm{D}_{6}, 298 \mathrm{~K}\right): \delta-110.14$ (br). ATR-IR v/cm ${ }^{-1}: 2939(\mathrm{w}), 2921(\mathrm{w})$, $2886(\mathrm{w}), 2860(\mathrm{~m}), 2763(\mathrm{w}), 1458(\mathrm{~m}), 1379(\mathrm{w}), 1359(\mathrm{w}), 1272(\mathrm{w}), 1235(\mathrm{w}), 1137(\mathrm{w})$, 1037 (m), 1009 (m), 926 (s), 880 (s), 806 (w), 730 (vs), 673 (s), 625 (m), $561(\mathrm{w}), 516(\mathrm{w})$, 442 (w). UV/Vis/NIR (THF, $298 \mathrm{~K}) \lambda_{\max } / \mathrm{cm}^{-1}\left(\varepsilon / \mathrm{dm}^{3} \mathrm{~mol}^{-1} \mathrm{~cm}^{-1}\right): 19083$ (42), 17794 (29), 15198 (34), 14084 (55), 12853 (18), 9124 (34), 7364 (15). $\mu_{\text {eff }}$ (Evan's method, $\mathrm{C}_{6} \mathrm{D}_{6}, 298 \mathrm{~K}$ ): $2.7 \mu_{\mathrm{B}}$

\section{Attempted Reaction of [(Tren $\left.\left.{ }^{\text {TIPS }}\right) U^{I I I}\right]$ (1) with Anthracene $e_{2}-P_{2}$}

A colorless solution of anthracene $2-\mathrm{P}_{2}(0.04 \mathrm{~g}, 0.1 \mathrm{mmol})$ in $\mathrm{C}_{6} \mathrm{D}_{6}(1.0 \mathrm{~mL})$ was added to a dark purple solution of $1(0.08 \mathrm{~g}, 0.1 \mathrm{mmol})$ in $\mathrm{C}_{6} \mathrm{D}_{6}(1.0 \mathrm{~mL})$. The mixture remained as a purple solution after the addition, and no color change was observed after 24 hours at room temperature. ${ }^{1} \mathrm{H}$ NMR of the solution showed that most of the anthracene $e_{2}-\mathrm{P}_{2}(>90 \%)$ had decomposed to anthracene and other unidentifiable products, with $\mathbf{1}$ and a very small amount anthracene $2-\mathrm{P}_{2}$ being left in the purple solution. No formation or precipitation of $\mathbf{5}$ was 
observed from the reaction (Figure S9). No formation of anthracene $2-\mathrm{P}_{2}$ observed in the reaction of 1 with excess 4 in $\mathrm{C}_{6} \mathrm{D}_{6}$ when monitored by an in-situ NMR study.

\section{Synthesis and Isolation of $\left[\left\{\left(\operatorname{Tren}^{T I P S}\right) U^{I V}\right\}_{2}\left(\mu-\eta^{3}: \eta^{3}-P_{3}\right)\right]\left[M(\text { arene })_{4}\right](7 M)$}

Representative procedure for $7 \boldsymbol{R} \boldsymbol{b}$. Finely ground 5 (34 mg, $0.02 \mathrm{mmol})$ and $\mathrm{RbC}_{8}(15 \mathrm{mg}$, $0.08 \mathrm{mmol})$ were weighed into a small glass vial with a stir bar, and then $\mathrm{d}_{6}$-benzene $(1 \mathrm{~mL})$ was added to form a red suspension. The mixture was stirred for 24 hours, turning into a dark red solution. The mixture was filtered into a J-Young NMR tube, and ${ }^{1} \mathrm{H}$ NMR spectroscopy of that filtrate revealed that the known cyclometallate complex $\left[\mathrm{U}^{\mathrm{IV}}\left\{\mathrm{N}\left(\mathrm{CH}_{2} \mathrm{CH}_{2} \mathrm{NSiPr}_{3}\right)_{2}\left(\mathrm{CH}_{2} \mathrm{CH}_{2} \mathrm{NSiPr}_{2}{ }_{2} \mathrm{CH}(\mathrm{Me})\left(\mathrm{CH}_{2}\right)\right\}\right] \quad(\mathbf{8})^{13}\right.$ is the main product. However, standing the sample in the NMR tube for 2 days produced a very small crop of dark red crystals, which were suitable for single-crystal XRD. Bulk scale reaction in benzene also produced a very small crop of dark red crystals of $\left[\left\{\left(\operatorname{Tren}^{\mathrm{TIPS}}\right) \mathrm{U}^{\mathrm{IV}}\right\}_{2}\left(\mu-\eta^{3}: \eta^{3}-\right.\right.$ $\left.\left.\left.\mathrm{P}_{3}\right)\right][\mathrm{Rb} \text { (benzene) })_{4}\right](\mathbf{7} \mathbf{R b})$, therefore the low yield prevented further characterization, but it could be reproducibly isolated as a crystalline solid in very low yield on each occasion. Dark red crystals of the $\left[\mathrm{K}(\text { toluene })_{4}\right]^{+}$and $\left[\mathrm{Cs}(\text { toluene })_{4}\right]^{+}$analogs were obtained by cooling the corresponding toluene solutions from $\mathrm{KC}_{8}$ and $\mathrm{CsC}_{8}$ reductions at $-30{ }^{\circ} \mathrm{C}$ for 2 days. The yield for the $\mathrm{Cs}$ analogue is slightly higher than those for $\mathrm{K}$ and $\mathrm{Rb}$ ones, but still $<5 \%$, nevertheless a small crop of pure crystalline solid was isolated for limited characterization. Anal. Calcd for $\mathrm{C}_{94} \mathrm{H}_{182} \mathrm{CsN}_{8} \mathrm{P}_{3} \mathrm{Si}_{6} \mathrm{U}_{2} \bullet$ (toluene): C, 50.82; H, 8.02; N, 4.69\%. Found: $\mathrm{C}, 50.33$; H, 7.98; N, 4.82\%. ATR-IR v/cm-1: 2919 (br, w), 2857 (br, m), 2832 (m), 1463 (m), 1386 (w), $1335(\mathrm{w}), 1277(\mathrm{w}), 1252(\mathrm{w}), 1146(\mathrm{w}), 1037(\mathrm{~m}), 1012(\mathrm{~m}), 935$ (s), $878(\mathrm{~s}), 792$ (s), 737 (vs), 668 (s), 618 (m), $571(\mathrm{w}), 515$ (w), 441 (w). Solution NMR spectra could not be obtained due to very poor solubility in toluene/benzene once isolated in pure crystalline form. 


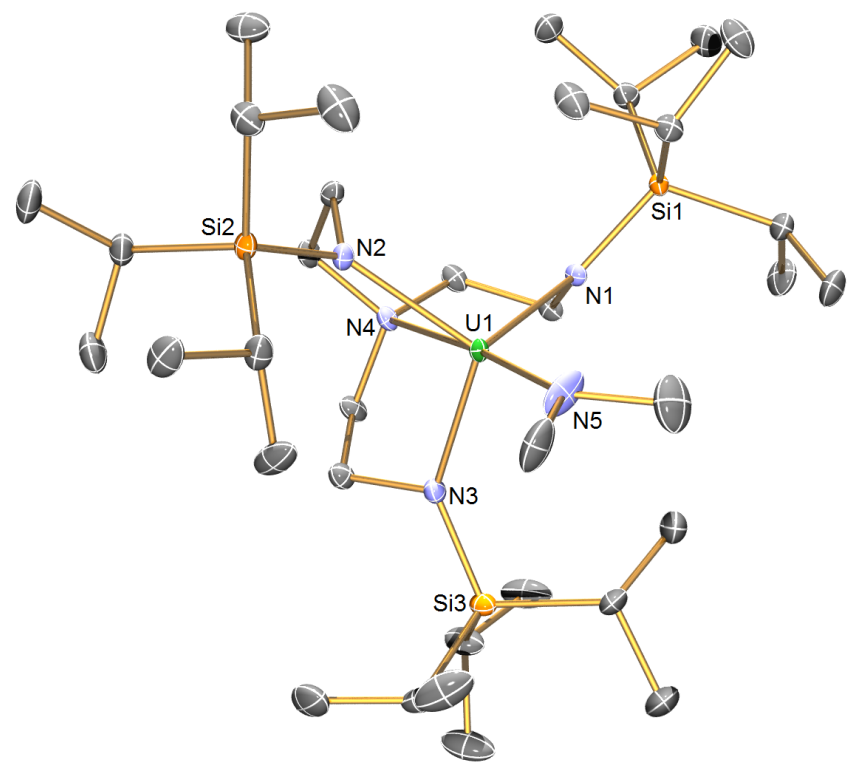

Figure S1. Molecular structure of 6 at $150 \mathrm{~K}$ with selected atom labels. Displacement ellipsoids are set at 40\% and hydrogen atoms and disordered components are omitted for clarity.
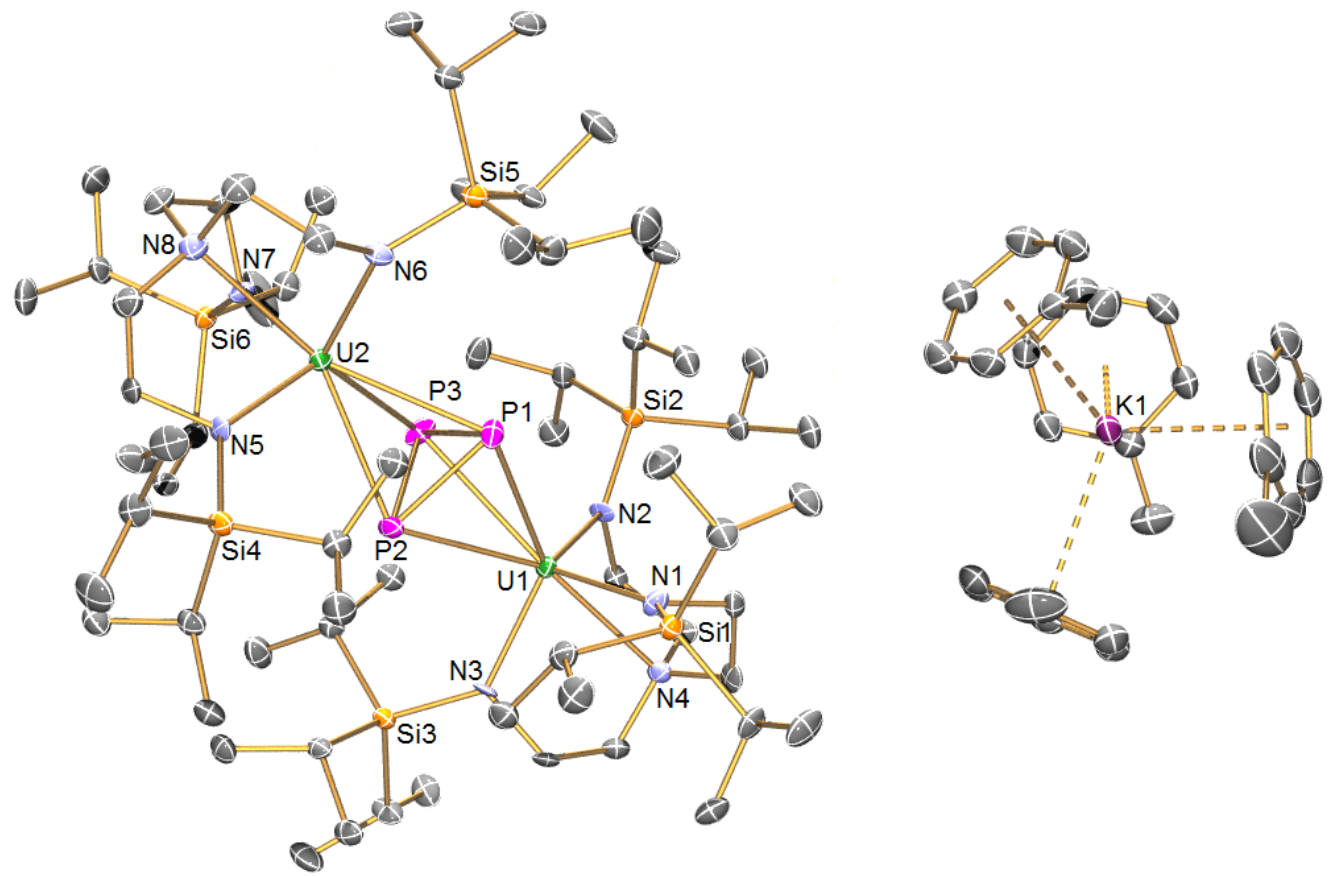

Figure S2. Molecular structure of $7 \mathrm{~K}$ at $120 \mathrm{~K}$ with selected atom labels. Displacement ellipsoids are set at $40 \%$ and hydrogen atoms, the toluene molecule in the lattice, and disorder components are omitted for clarity. 

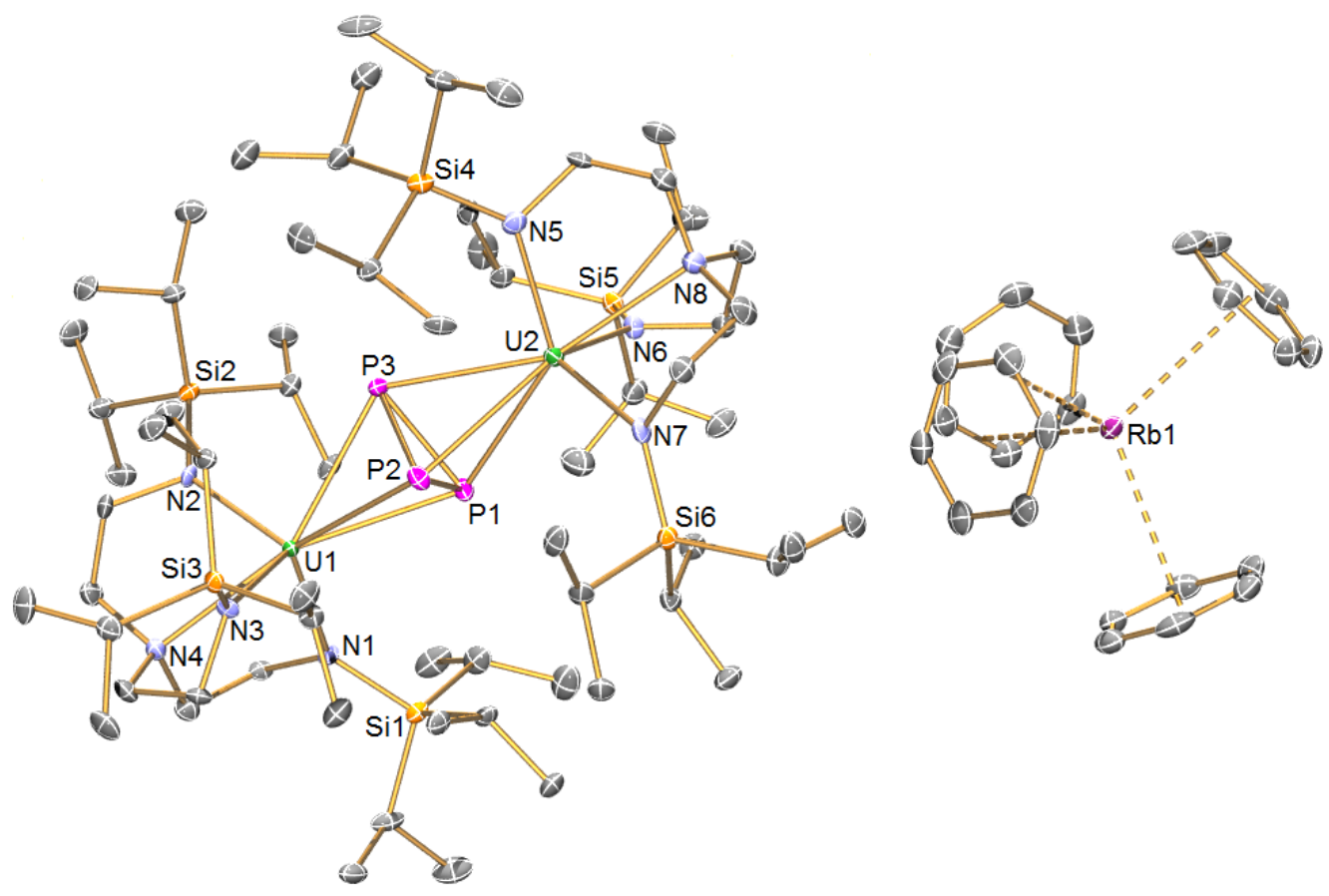

Figure S3. Molecular structure of $7 \mathbf{R b}$ at $150 \mathrm{~K}$ with selected atom labels. Displacement ellipsoids are set at $40 \%$ and hydrogen atoms, the benzene molecule in the lattice, and disorder components are omitted for clarity.
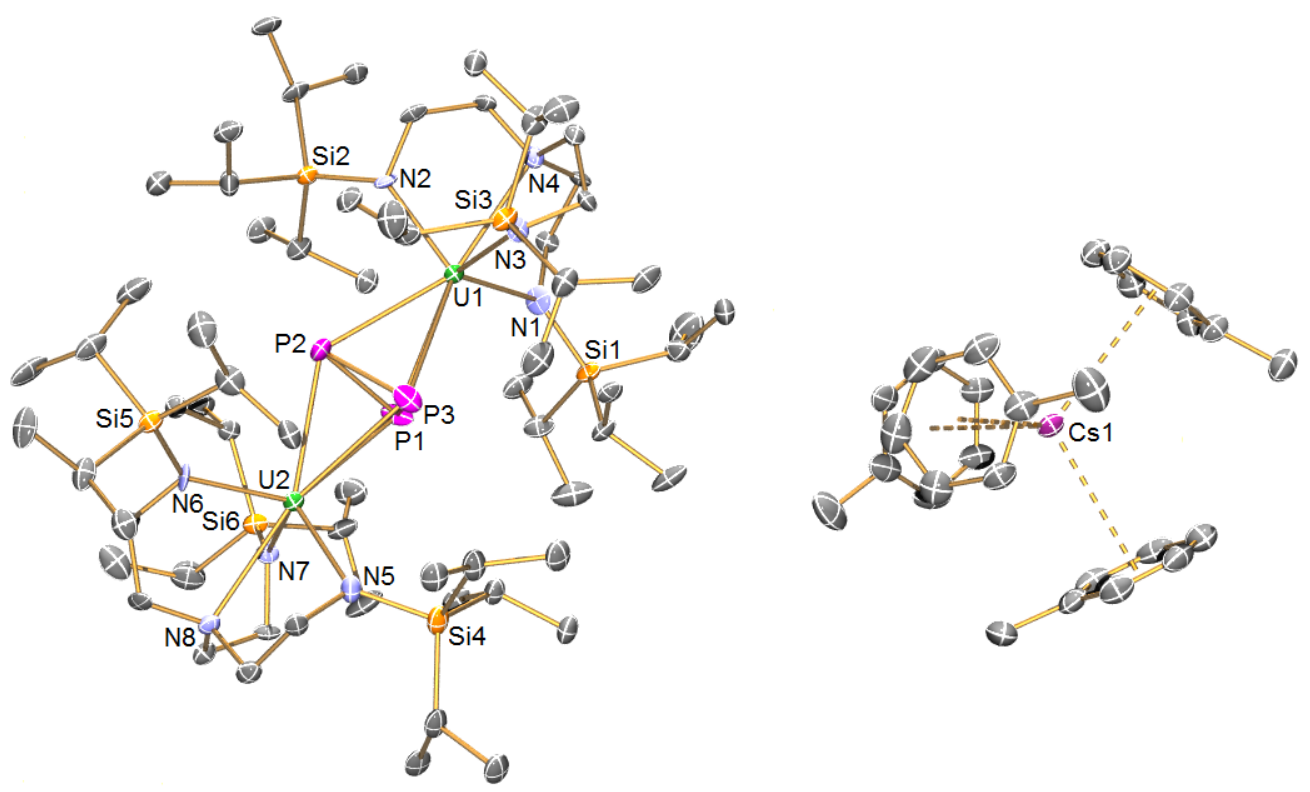

Figure S4. Molecular structure of $7 \mathrm{Cs}$ at $100 \mathrm{~K}$ with selected atom labels. Displacement ellipsoids are set at $40 \%$ and hydrogen atoms, the toluene molecule in the lattice, and disorder components are omitted for clarity. 
Spectroscopic and Magnetic Data

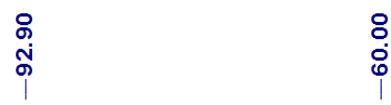

$\stackrel{8}{\circ}$

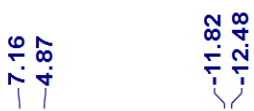

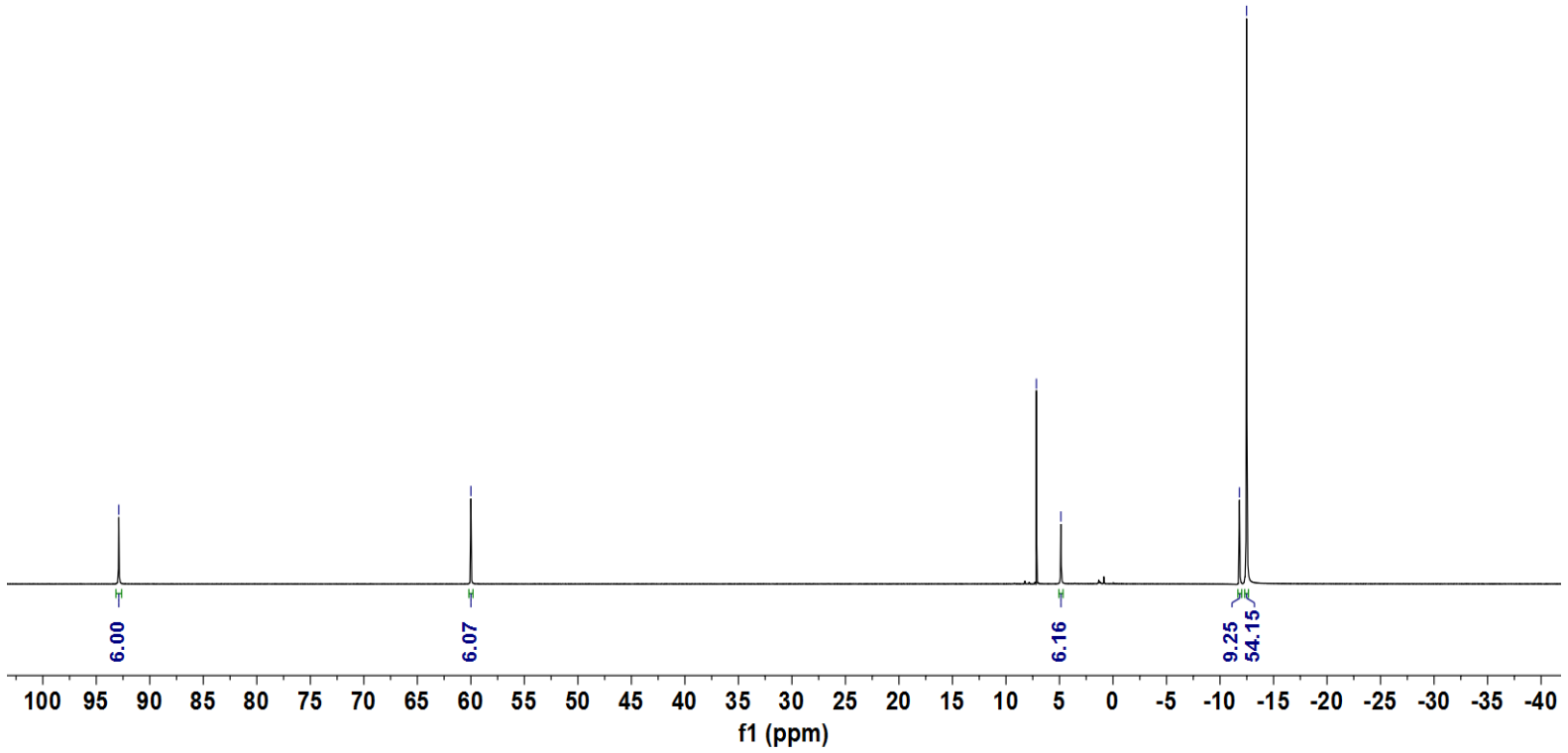

Figure S5. ${ }^{1} \mathrm{H}$ NMR (400 MHz, $\left.\mathrm{C}_{6} \mathrm{D}_{6}, 298 \mathrm{~K}\right)$ of 6.

$\frac{i}{i}$

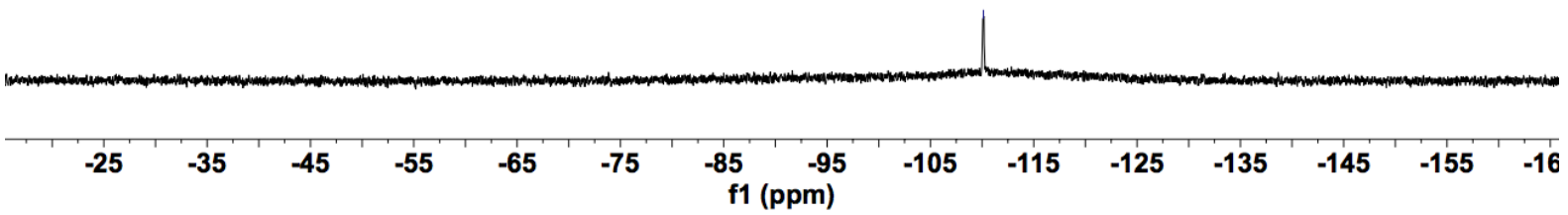

Figure S6. ${ }^{1} \mathrm{H}$ NMR (79 MHz, $\left.\mathrm{C}_{6} \mathrm{D}_{6}, 298 \mathrm{~K}\right)$ of 6. 


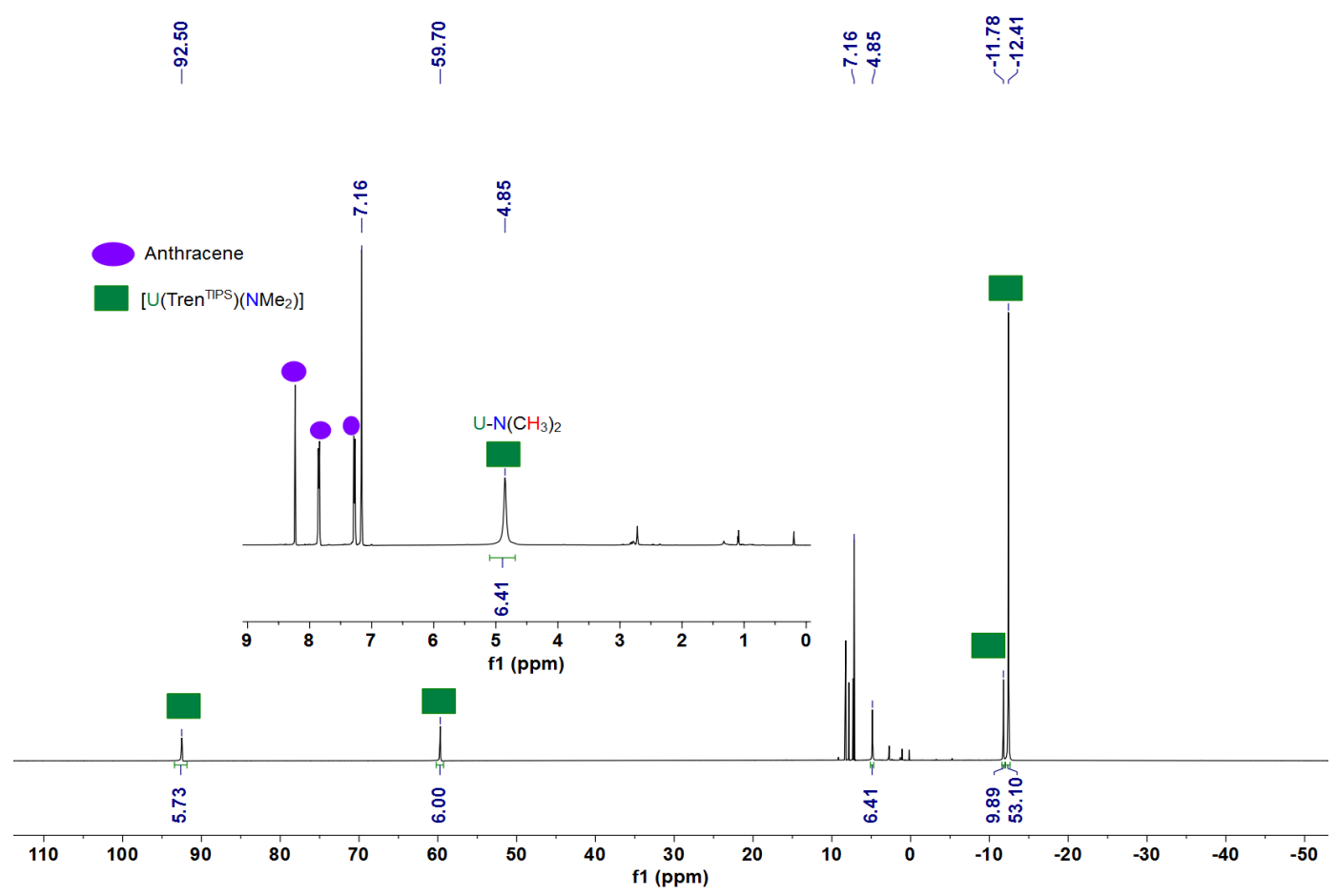

Figure S7. ${ }^{1} \mathrm{H}$ NMR spectrum of the solution phase products (anthracene and 6) for the reaction of $\mathbf{1}$ and $\mathbf{4}$ in $\mathrm{C}_{6} \mathrm{D}_{6}$.

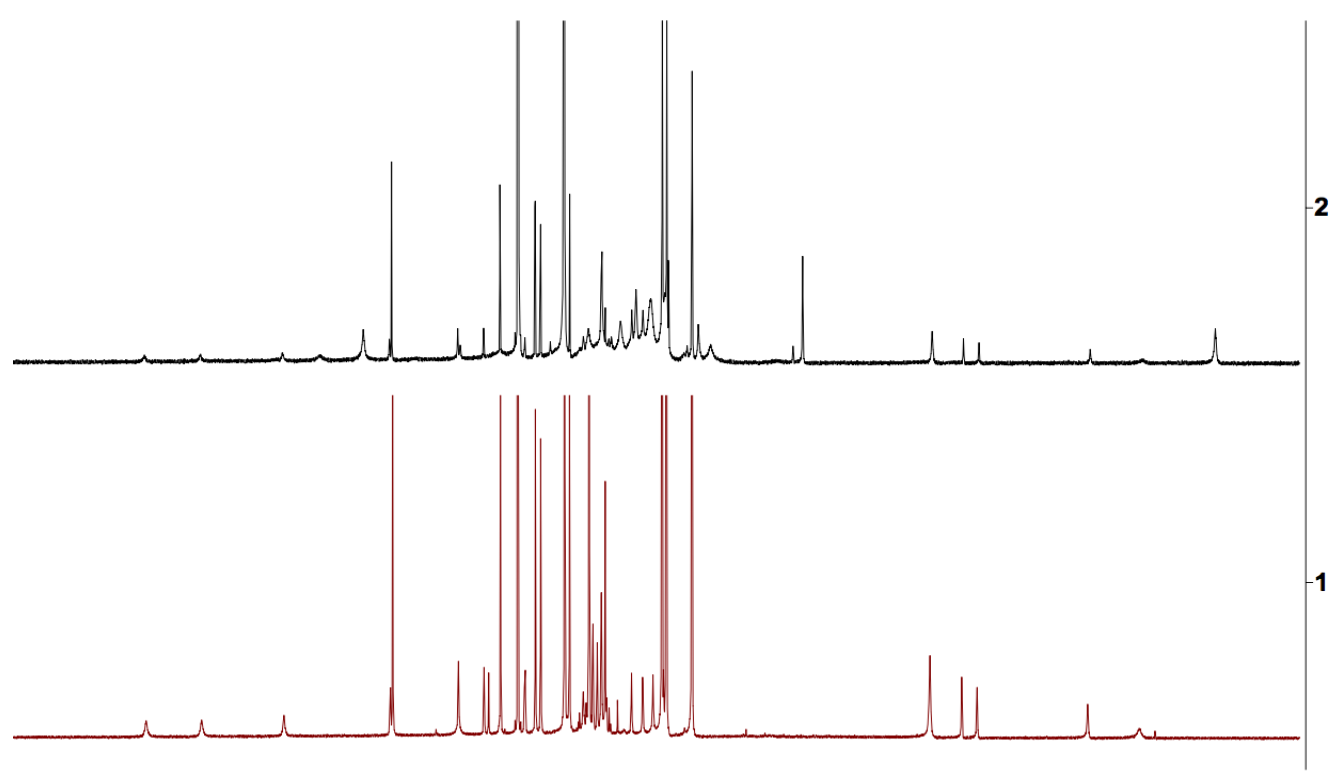

\begin{tabular}{lllllllllllllllllll}
\hline 40 & 35 & 30 & 25 & 20 & 15 & 10 & 5 & $\begin{array}{r}0 \\
\mathrm{f} 1(\mathrm{ppm})\end{array}$ & -10 & -15 & -20 & -25 & -30 & -35 & -40 & -45
\end{tabular}

Figure S8. ${ }^{1} \mathrm{H}$ NMR spectra of the reduction of 5 with $2.0 \mathrm{RbC}_{8}$ in $\mathrm{C}_{6} \mathrm{D}_{6}$ (top, black) and $\mathbf{8}$ (bottom, red). 

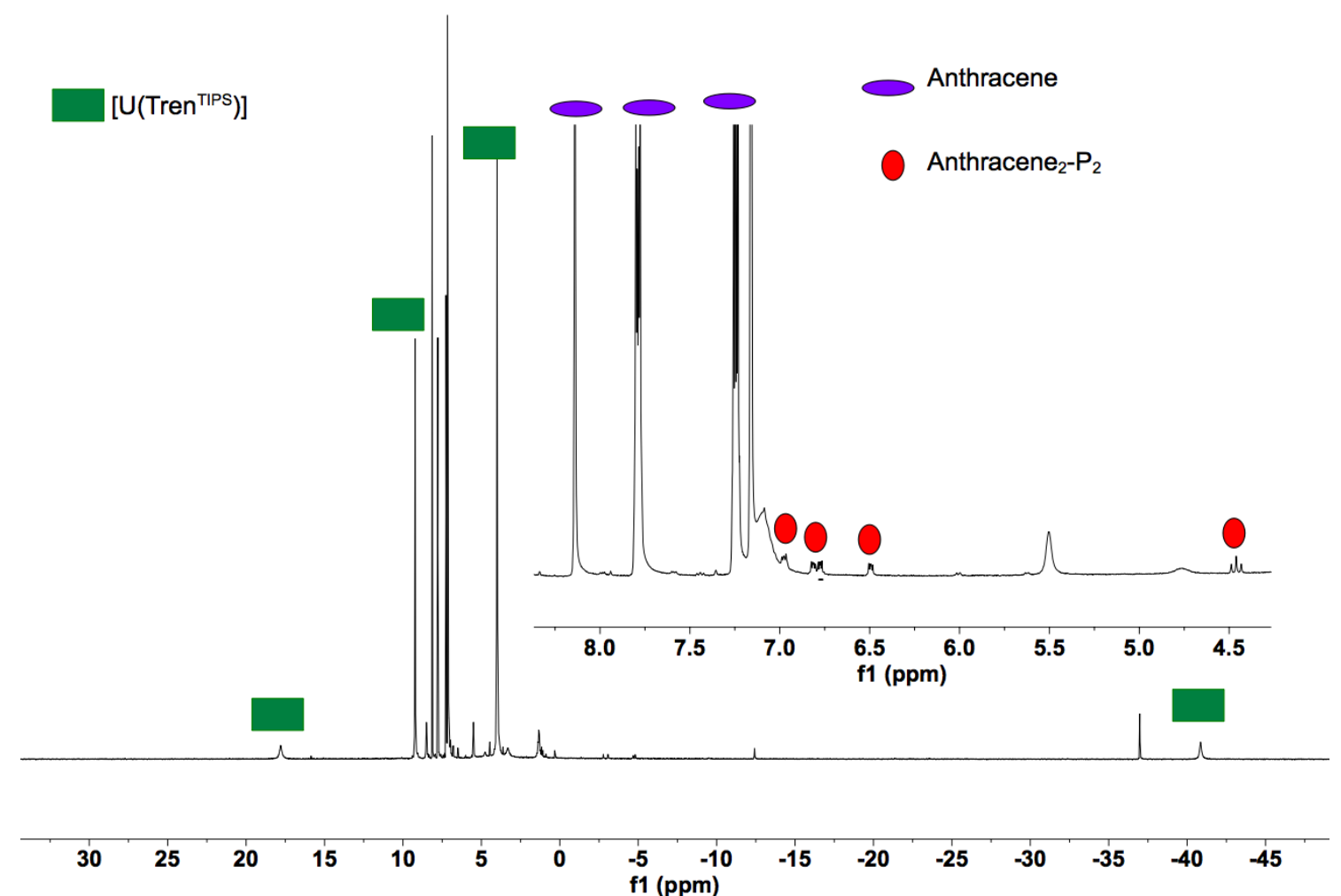

Figure S9. ${ }^{1} \mathrm{H}$ NMR spectra of the reaction of 1 with anthracene $2-\mathrm{P}_{2}$ in $\mathrm{C}_{6} \mathrm{D}_{6}$.

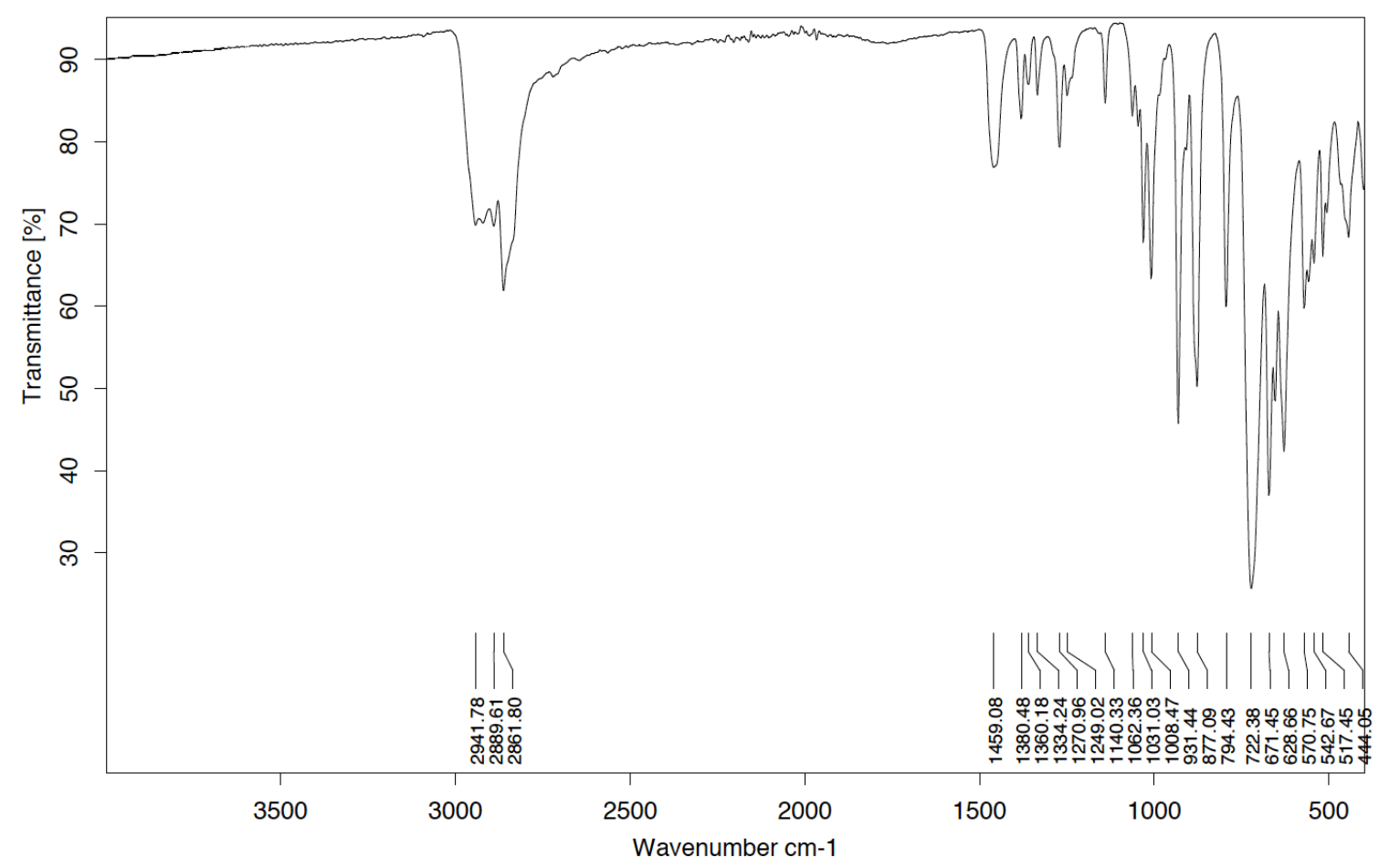

Figure S10. ATR-IR spectrum of 5. 


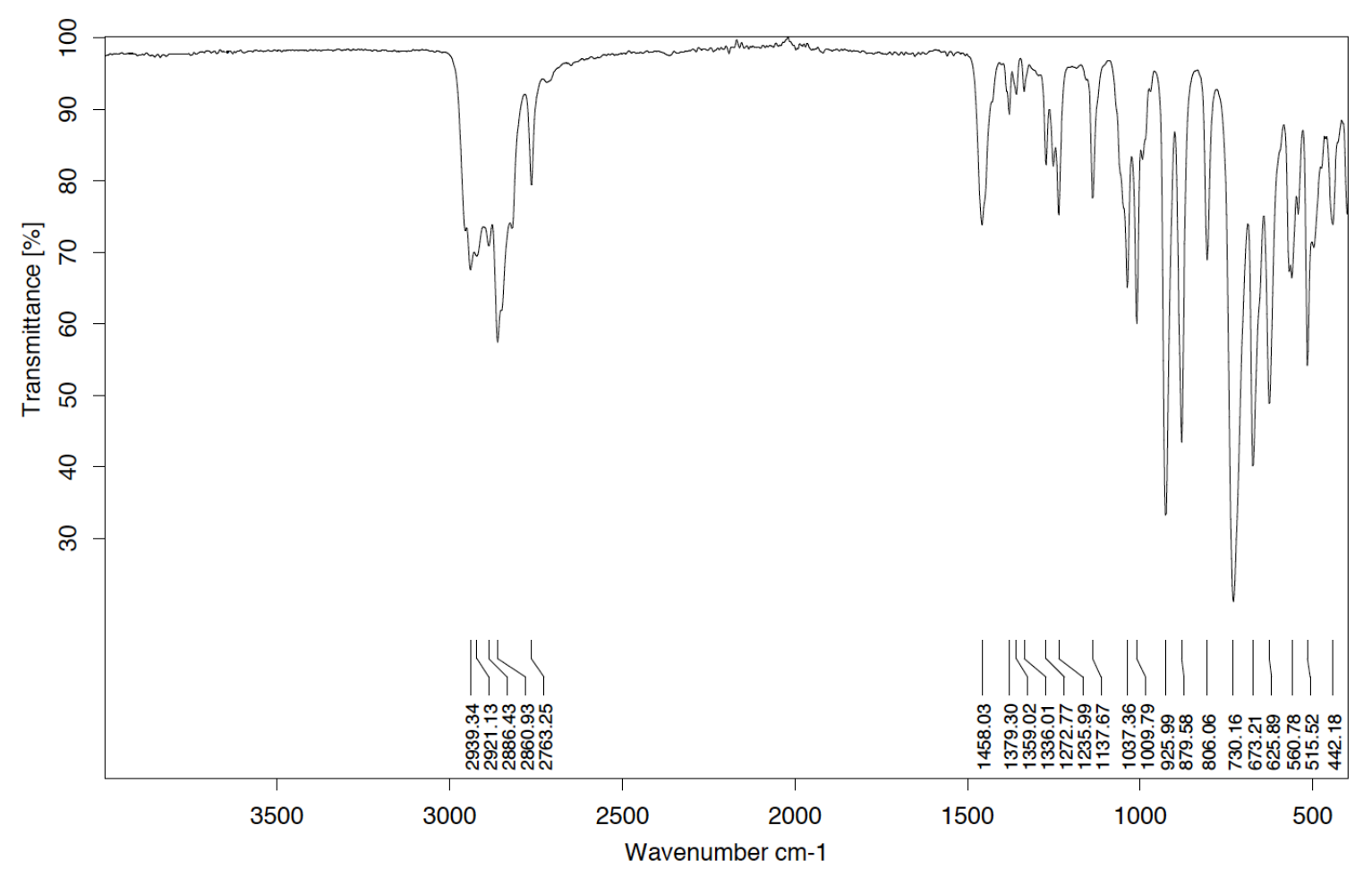

Figure S11. ATR-IR spectrum of 6.

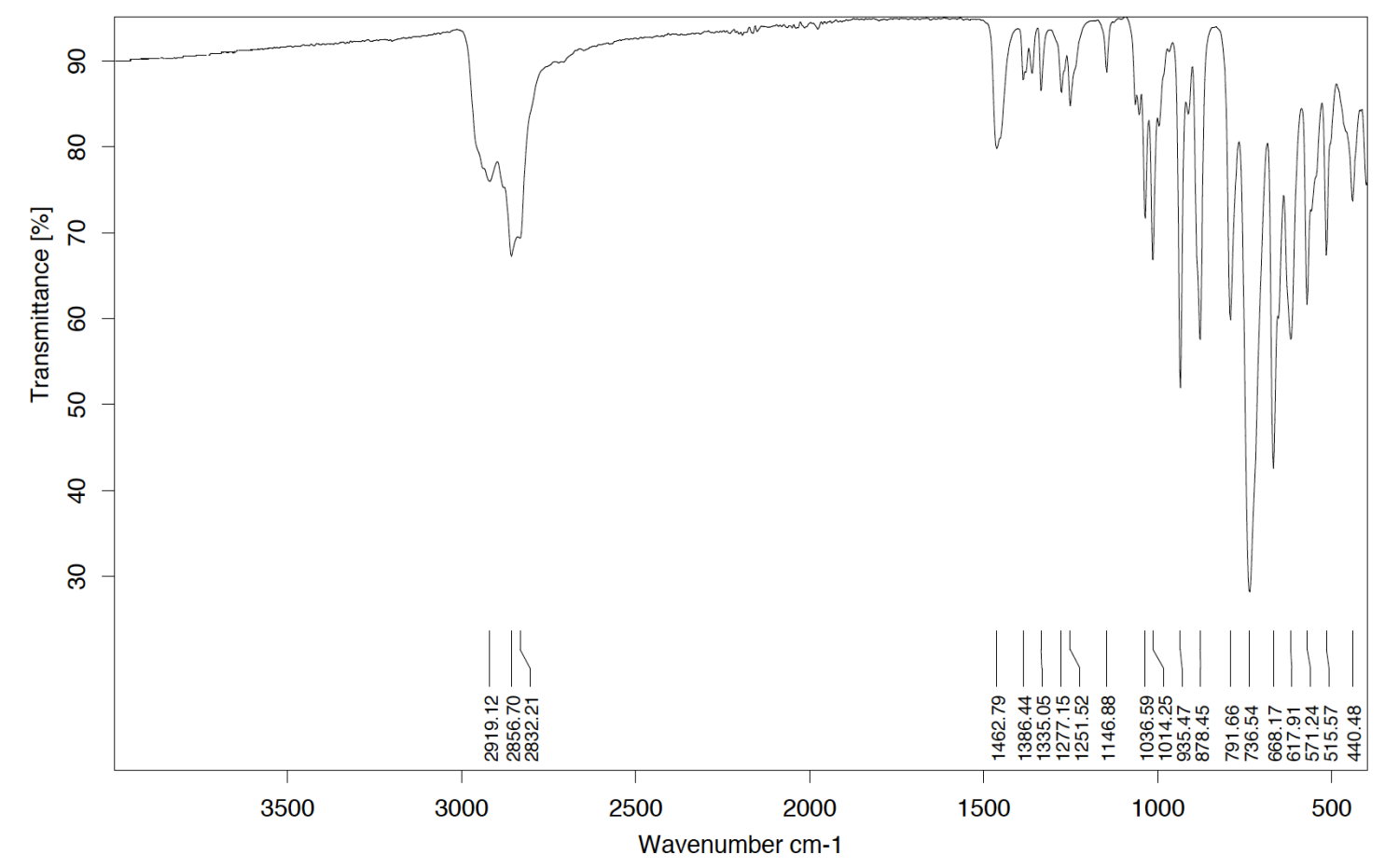

Figure S12. ATR-IR spectrum of 7Cs. 

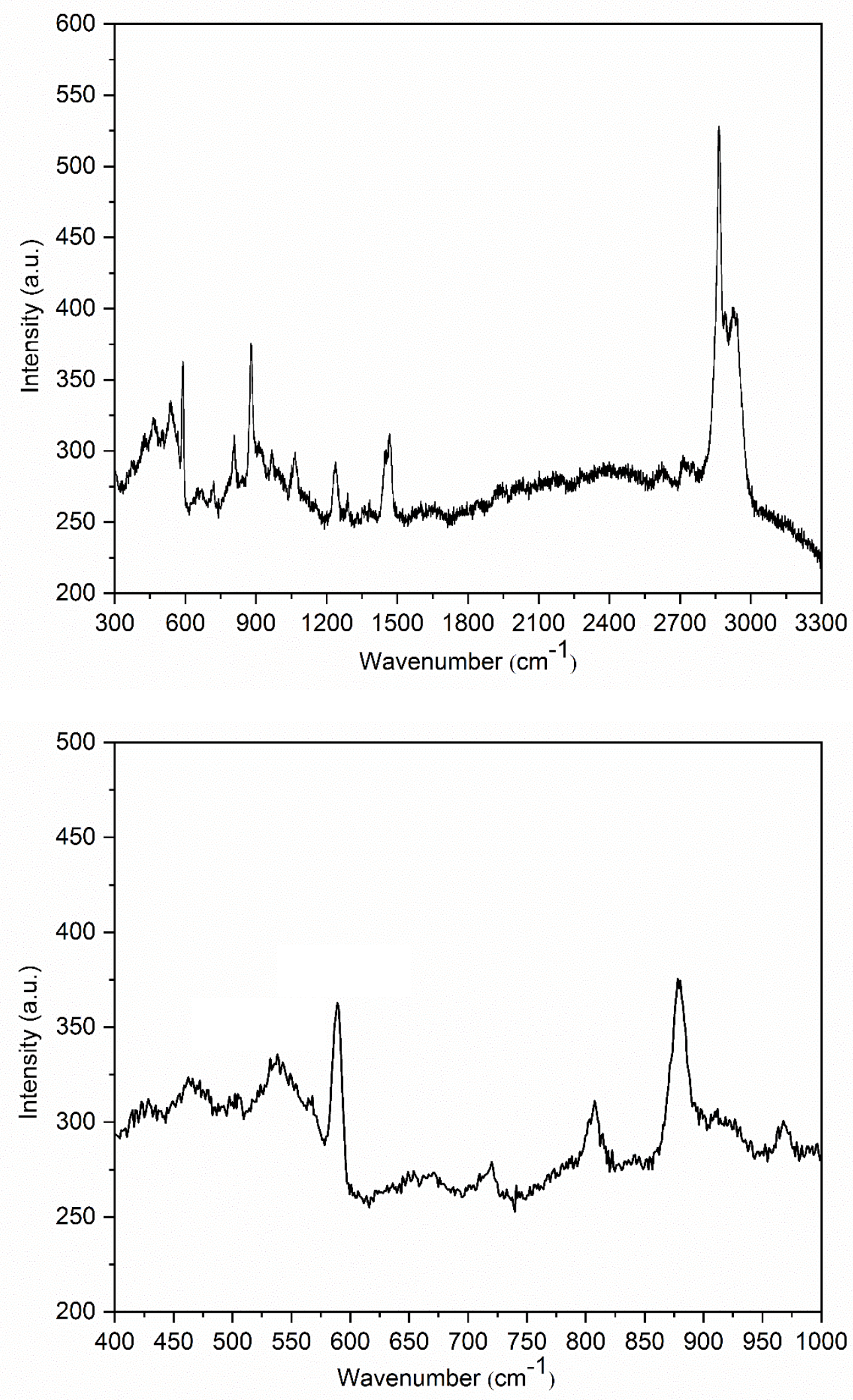

Figure S13. Raman spectra of complex 5 recorded at crystalline solid samples (top: 300 to $3300 \mathrm{~cm}^{-1}$; bottom: 400 to $1000 \mathrm{~cm}^{-1}$ ). 


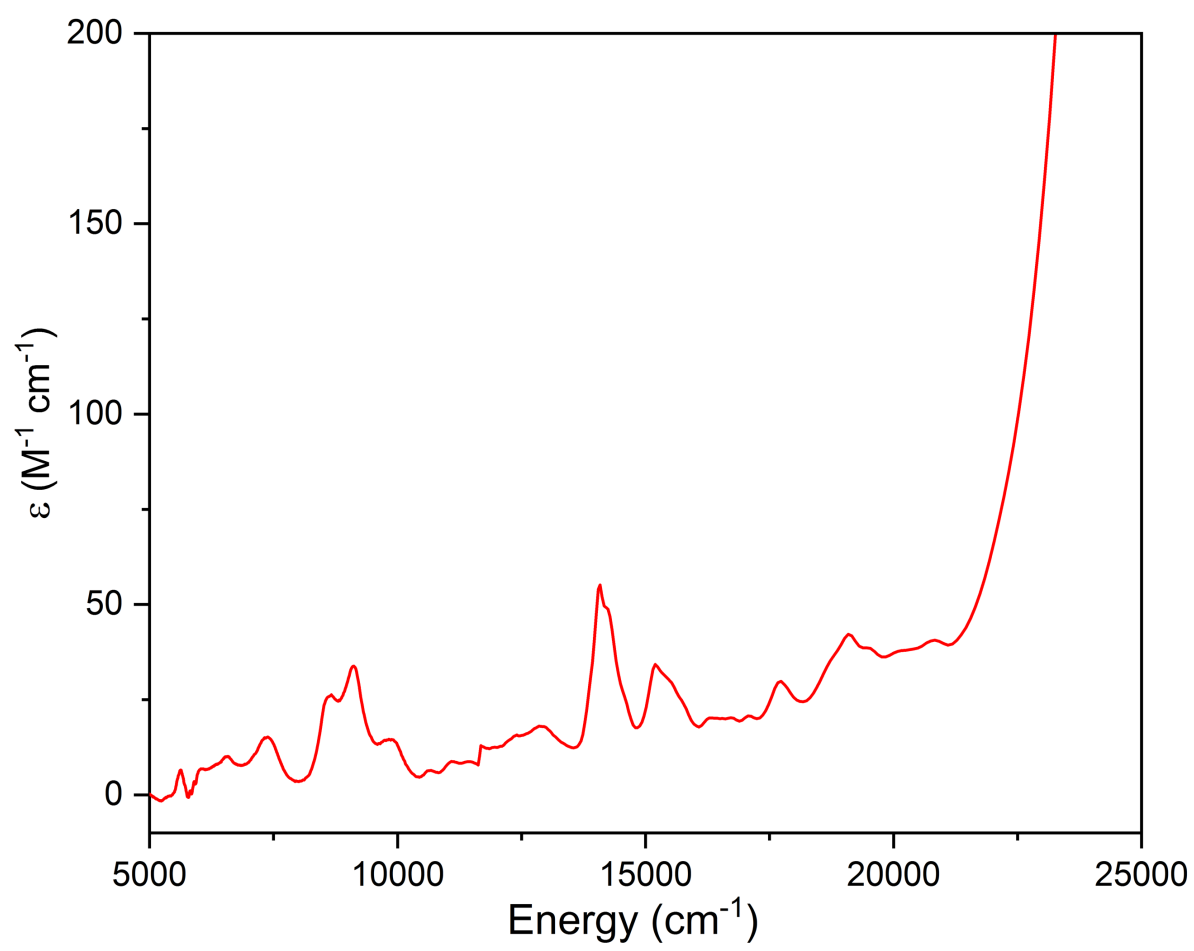

Figure S14. UV/Vis/NIR spectrum of 6 in THF over the range $5000-50000 \mathrm{~cm}^{-1}$.
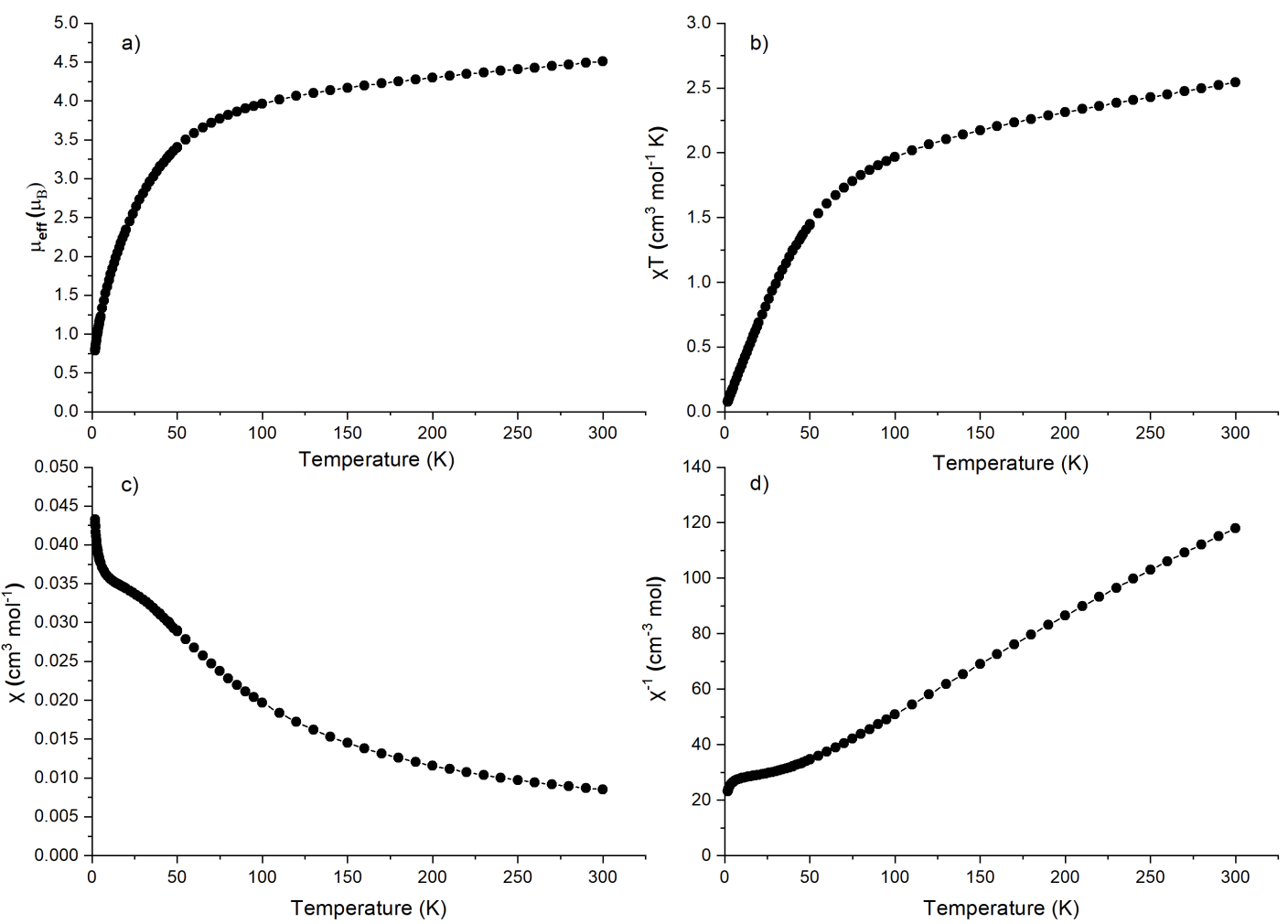

Figure S15. Variable-temperature SQUID magnetometry of 5 (whole molecule) over the temperature range $1.8-300 \mathrm{~K}$ : a) $\mu_{\text {eff }} \mathrm{vs} \mathrm{T}$; b) $\chi \mathrm{T}$ vs $\mathrm{T}$; c) $\chi$ vs T; d) $\chi^{-1}$ vs $\mathrm{T}$. 


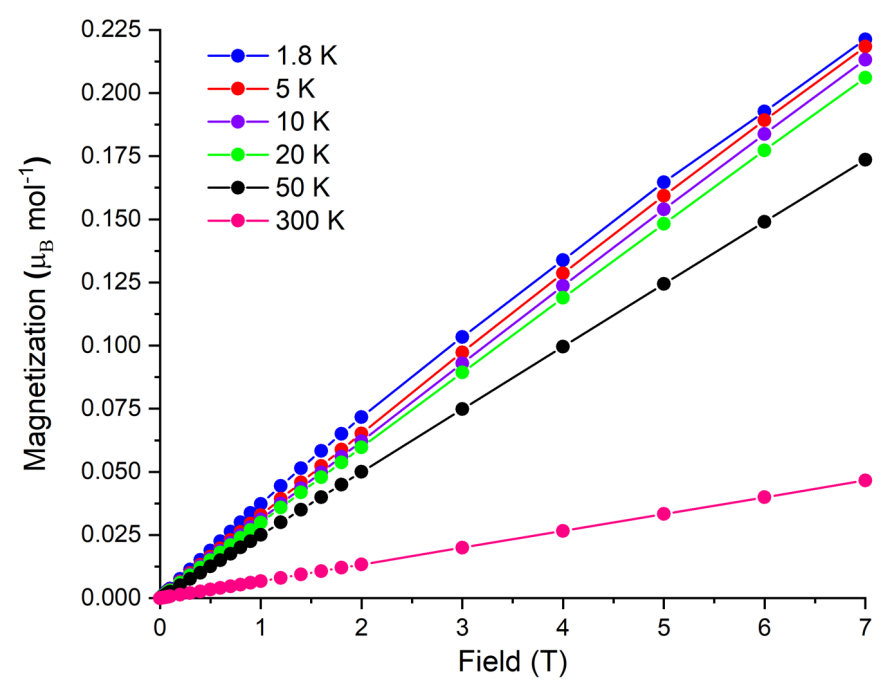

Figure S16. Magnetization vs Field data for 5 at 1.8, 5, 10, 20, 50, and $300 \mathrm{~K}$.

\section{Theoretical Calculations}

\section{General}

Geometry optimizations for 5 and the anion component of $\mathbf{7 M}, \mathbf{7}^{\prime}, \mathrm{N}_{2}$, and $\mathrm{P}_{2}$ were performed using coordinates derived from the crystal structures of $\mathbf{5}, \mathbf{7} \mathbf{R b}$, and twice the pnictogen covalent radius, respectively, as the starting points. No constraints were imposed on the structures during the geometry optimizations. The calculations were performed using the Amsterdam Density Functional (ADF) suite version 2017 with standard convergence criteria. ${ }^{14,15}$ The DFT geometry optimizations employed Slater type orbital (STO) triple- $\zeta-$ plus polarization all electron basis sets (from the Dirac and ZORA/TZP database of the ADF suite). Scalar relativistic approaches (spin-orbit neglected) were used within the ZORA Hamiltonian ${ }^{16-18}$ for the inclusion of relativistic effects and the local density approximation (LDA) with the correlation potential due to Vosko et al was used in all of the calculations. ${ }^{19}$ Generalized gradient approximation corrections were performed using the functionals of Becke and Perdew. ${ }^{20,21}$ MOLEKEL $^{22}$ was used to prepare the three-dimensional plots of the electron density. Frequencies were computed using the analytical frequencies routine in ADF. 

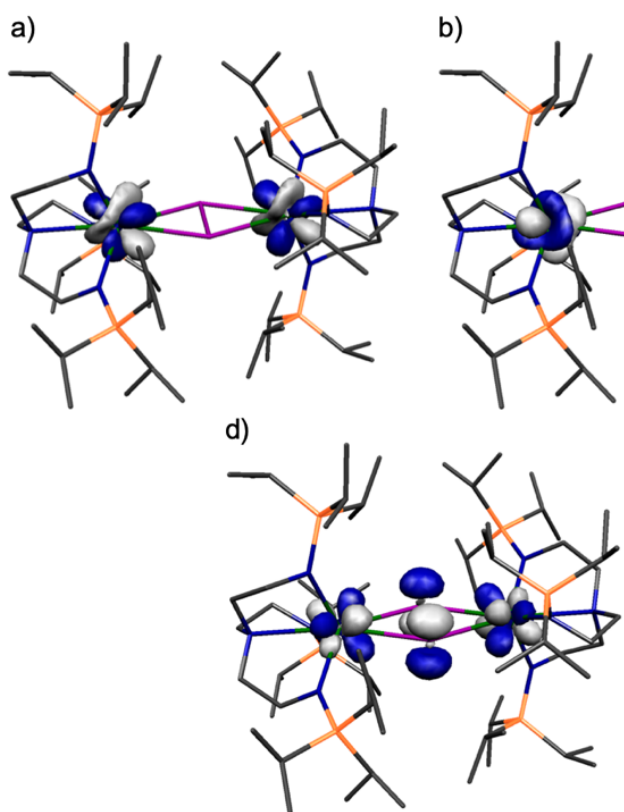

b)

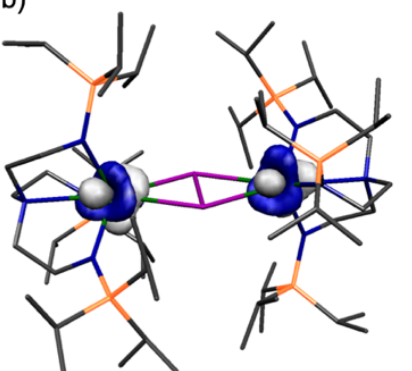

.

. c) $1 /$

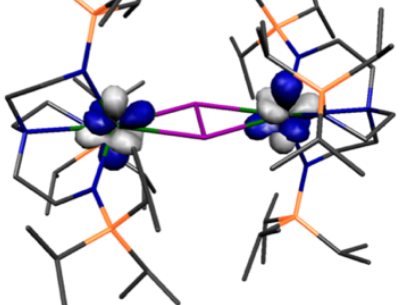

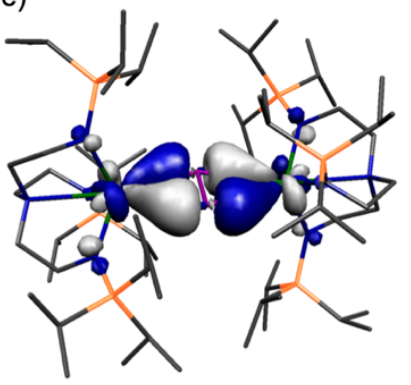

Figure S17. Selected Kohn Sham Molecular Orbitals of 5quin: a) HOMO (452a, $-3.198 \mathrm{eV}$ ), b) HOMO-1 (451a, -3.242 eV), c) HOMO-2 (450a, -3.249 eV), d) HOMO-3 (449a, -3.563 eV), e) HOMO-4 (448a, $-4.314 \mathrm{eV})$. Hydrogen atoms are omitted for clarity.

.

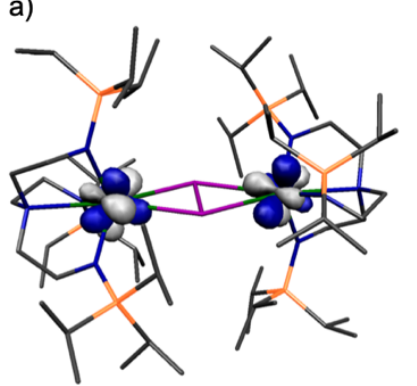

d)

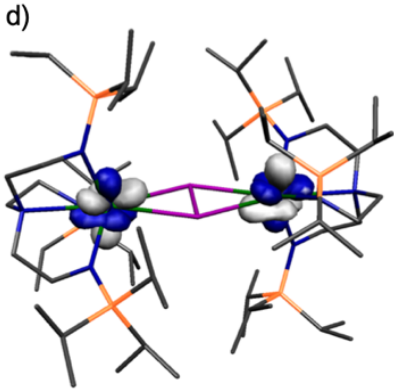

D)

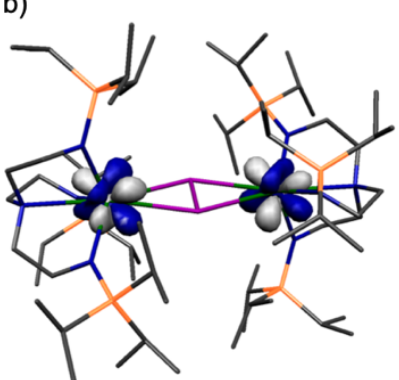

.

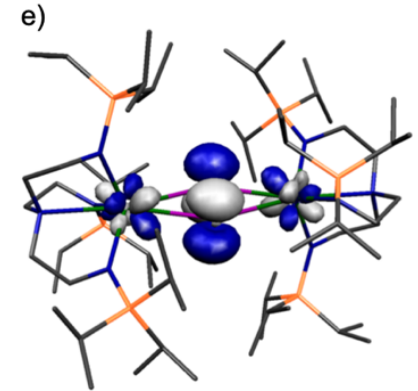

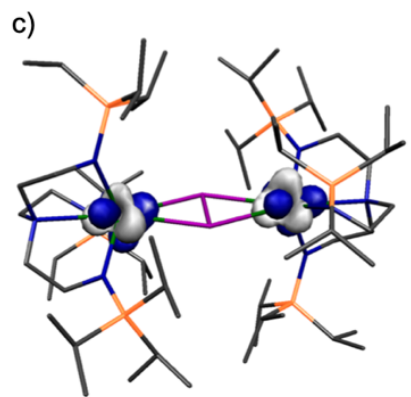

.

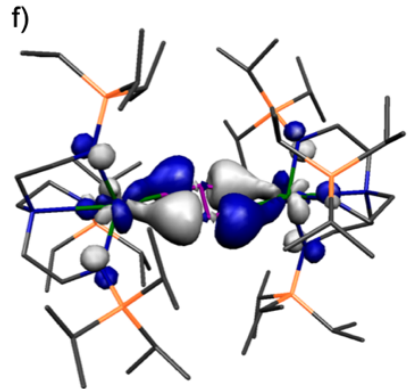

Figure S18. Selected Kohn Sham Molecular Orbitals of 5sept: a) HOMO (453a, -2.976 eV), b) HOMO-1 (452a, -2.991 eV), c) HOMO-2 (451a, -2.994 eV), d) HOMO-3 (450a, -3.051 eV), e) HOMO-4 (449a, -3.577 eV), f) HOMO-5 (448a, -4.480 eV). Hydrogen atoms are omitted for clarity. 
a)

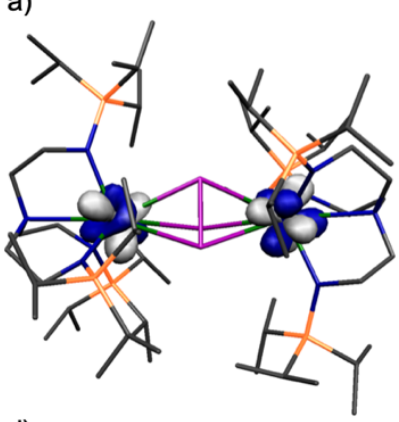

d)

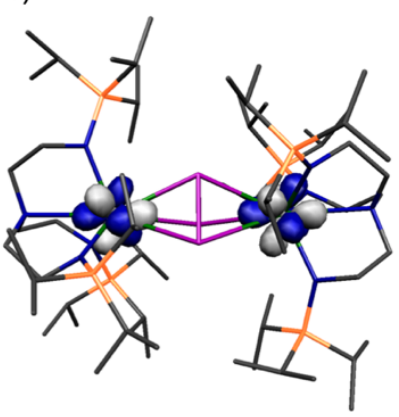

b)

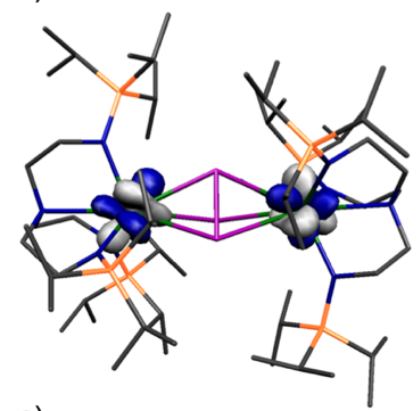

e)

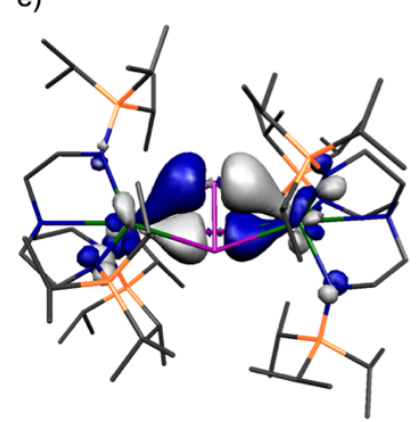

c)

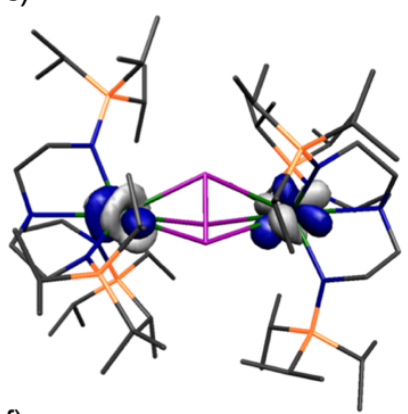

f)

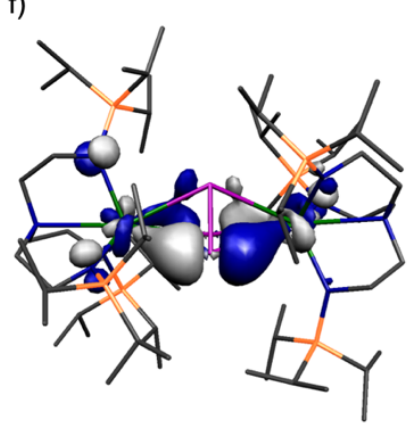

Figure S19. Selected Kohn Sham Molecular Orbitals of 7': a) HOMO (460a, $-0.073 \mathrm{eV})$, b) HOMO-1 (459a, -0.083 eV), c) HOMO-2 (458a, -0.086 eV), d) HOMO-3 (457a, -0.109 eV), e) HOMO-4 (456a, -1.688 eV), f) HOMO-5 (455a, -1.713 eV). Hydrogen atoms are omitted for clarity.

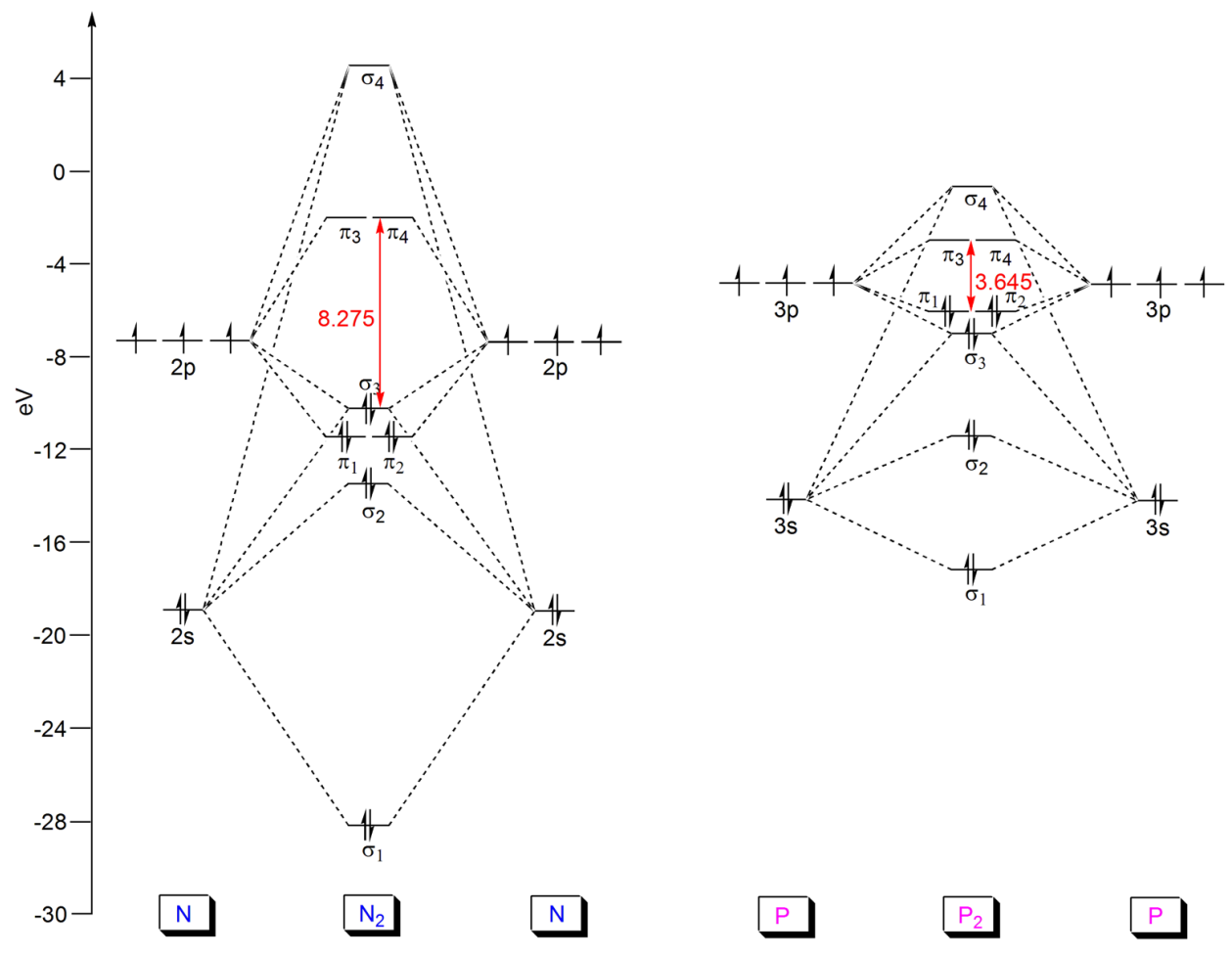

Figure S20. Molecular orbital energy levels for geometry optimized $\mathrm{N}_{2}$ and $\mathrm{P}_{2}$. 
Table S1. Experimental and Computed Data for 5, 5quin, and 5sept.

\begin{tabular}{|c|c|c|c|c|c|c|}
\hline \multicolumn{7}{|c|}{5} \\
\hline U-P & $2.9466(12)$ & $2.9466(12)$ & $2.9441(12)$ & $2.9441(12)$ & & \\
\hline U-N $\mathrm{N}_{\text {amide }}$ & $2.273(3)$ & $2.273(3)$ & $2.271(3)$ & $2.271(3)$ & $2.267(3)$ & $2.267(3)$ \\
\hline U-N $\mathrm{N}_{\text {amine }}$ & $2.690(4)$ & $2.690(4)$ & & & & \\
\hline $\mathrm{U} \bullet \bullet \bullet U$ & $5.528(3)$ & & & & & \\
\hline \multicolumn{7}{|c|}{ 5quin } \\
\hline U-P & 2.9456 & 2.9456 & 2.9177 & 2.9176 & & \\
\hline U-N $\mathrm{N}_{\text {amide }}$ & 2.298 & 2.290 & 2.287 & 2.298 & 2.290 & 2.287 \\
\hline U-N amine & 2.778 & 2.778 & & & & \\
\hline $\mathrm{U} \bullet \bullet \bullet$ & 5.484 & & & & & \\
\hline \multicolumn{7}{|c|}{5 sept } \\
\hline U-P & 3.1409 & 3.1390 & 2.9570 & 2.9545 & & \\
\hline U-N $\mathrm{N}_{\text {amide }}$ & 2.301 & 2.301 & 2.300 & 2.300 & 2.290 & 2.290 \\
\hline U-N $\mathrm{N}_{\text {amine }}$ & 2.730 & 2.730 & & & & \\
\hline U•••U & 5.747 & & & & & \\
\hline
\end{tabular}

Table S2. Final Coordinates and Energy for Single Point Energy Calculation on Geometry

\section{Optimized $P_{2}$}

$$
\begin{array}{llll}
1 . P & 0.000000 & 0.000000 & 0.065989 \\
\text { 2.P } & 0.000000 & 0.000000 & 1.974011
\end{array}
$$

Energy: $-8.73681732 \mathrm{eV}$

Table S3. Final Coordinates and Energy for Single Point Energy Calculation on Geometry Optimized $\mathrm{P}_{2} \mathrm{H}_{2}$

$$
\begin{array}{crrr}
1 . \mathrm{H} & -0.501161 & 0.862700 & -1.516691 \\
2 . \mathrm{H} & 0.501161 & -0.862701 & 1.516691 \\
\text { 3.P } & -0.534047 & 0.869859 & -0.077579 \\
\text { 4.P } & 0.534047 & -0.869860 & 0.077579 \\
\text { Energy: } & -15.91992204 \mathrm{eV} &
\end{array}
$$

Table S4. Final Coordinates and Energy for Single Point Energy Calculation on Geometry Optimized $\mathrm{P}_{2}^{2-}$

$$
\begin{array}{cccr}
1 . P & 0.000000 & 0.000000 & -1.068733 \\
\text { 2.P } & 0.000000 & 0.000000 & 1.068733 \\
\text { Energy: } & -5.08397134 \mathrm{eV}
\end{array}
$$


Table S5. Final Coordinates and Energy for Single Point Energy Calculation on Geometry Optimized $\mathrm{P}_{2} \mathrm{H}_{4}$

$\begin{array}{llll}\text { 1.P } & -4.725621 & -4.088541 & 0.677253 \\ \text { 2.P } & -2.492453 & -4.402630 & 0.739758 \\ \text { 3.H } & -2.385797 & -4.593904 & -0.673436 \\ \text { 4.H } & -5.142726 & -5.451640 & 0.546759 \\ \text { 5.H } & -2.470453 & -5.810130 & 1.000332 \\ 6 . \mathrm{H} & -4.865437 & -4.109977 & 2.100202\end{array}$

Energy: $-23.72764647 \mathrm{eV}$

Table S6. Final Coordinates and Energy for Single Point Energy Calculation on Geometry

Optimized 5 (spin quintet)

$\begin{array}{lrrr}\text { 1.C } & 0.524313 & 0.310384 & -6.815073 \\ \text { 2.C } & 4.207870 & 0.162906 & -6.548961 \\ \text { 3.C } & 3.445079 & -0.896066 & -5.725506 \\ \text { 4.C } & 1.249832 & 2.344520 & -5.543581 \\ \text { 5.C } & 4.426848 & -1.986102 & -5.256454 \\ \text { 6.C } & 0.902976 & 0.848729 & -5.418793 \\ \text { 7.C } & 0.444558 & -2.546901 & -4.714482 \\ \text { 8.C } & -2.930873 & 1.956296 & -4.228587 \\ \text { 9.C } & -6.462013 & 2.631621 & -3.369987 \\ \text { 10.C } & 1.195783 & -1.697414 & -3.670885 \\ \text { 11.C } & 3.835272 & 1.712455 & -3.461649 \\ \text { 12.C } & -6.260303 & 0.155348 & -2.998908 \\ \text { 13.C } & -3.025888 & -4.254940 & -3.092742 \\ \text { 14.C } & 5.216693 & 1.286208 & -2.975751 \\ \text { 15.C } & -2.715073 & -0.259263 & -3.038352 \\ \text { 16.C } & -2.843715 & 1.262248 & -2.854569 \\ \text { 17.C } & 2.002544 & -2.623223 & -2.743318 \\ \text { 18.C } & -6.008389 & 1.541253 & -2.374713 \\ \text { 19.C } & -0.923090 & -5.498938 & -2.438507 \\ \text { 20.C } & 1.068314 & 3.969454 & -2.297462 \\ \text { 21.C } & -1.883538 & -4.353795 & -2.062836 \\ \text { 22.C } & 4.126613 & 5.879665 & -1.606640 \\ \text { 23.C } & -4.063822 & 3.722619 & -1.417299 \\ \text { 24.C } & 0.505877 & 6.047122 & -1.012843 \\ \text { 25.C } & 6.001222 & 0.018406 & -1.003462 \\ \text { 26.C } & -2.653770 & 4.146967 & -0.982921 \\ \text { 27.C } & -3.574740 & -7.010038 & -0.577426 \\ \text { 28.C } & 5.516695 & 2.423298 & -0.795962 \\ \text { 29.C } & 5.107330 & -1.206910 & -0.851038 \\ \text { 30.C } & 0.938053 & 4.569928 & -0.891891 \\ \text { 31.C } & -5.107164 & 4.386765 & -0.495829 \\ \text { 32.C } & -4.584822 & -2.554436 & -0.405898 \\ \text { 33.C } & 3.823944 & 5.651083 & -0.111060\end{array}$




\begin{tabular}{|c|c|c|c|}
\hline $34 . C$ & -3.823921 & -5.651215 & 0.110945 \\
\hline 35.C & 5.106834 & -4.386717 & 0.495642 \\
\hline 36. C & 4.585053 & 2.554587 & 0.406245 \\
\hline 37.C & 3.574886 & 7.009874 & 0.577467 \\
\hline 38.C & -5.516527 & -2.423209 & 0.796359 \\
\hline 39.C & -5.107642 & 1.207048 & 0.851154 \\
\hline 40.C & -6.001340 & -0.018371 & 1.003821 \\
\hline 41.C & -0.937975 & -4.570292 & 0.891896 \\
\hline 42.C & -0.505617 & -6.047439 & 1.012826 \\
\hline 43.C & 2.653511 & -4.146924 & 0.983213 \\
\hline 44.C & -4.126725 & -5.879621 & 1.606526 \\
\hline 45.C & 4.063648 & -3.722595 & 1.417345 \\
\hline 46.C & 1.883479 & 4.353846 & 2.062876 \\
\hline 47.C & 6.008343 & -1.541367 & 2.374621 \\
\hline 48.C & 0.922964 & 5.499047 & 2.438175 \\
\hline 49.C & -1.068302 & -3.969865 & 2.297492 \\
\hline 50.C & -2.003053 & 2.622900 & 2.742821 \\
\hline 51.C & 2.843704 & -1.262308 & 2.854901 \\
\hline 52.C & -5.216455 & -1.286044 & 2.976087 \\
\hline 53.C & 6.260435 & -0.155484 & 2.998789 \\
\hline 54.C & 3.025719 & 4.255136 & 3.092912 \\
\hline 55.C & 2.714963 & 0.259214 & 3.038537 \\
\hline 56.C & 6.461999 & -2.631795 & 3.369815 \\
\hline 57.C & -3.835065 & -1.712424 & 3.461699 \\
\hline 58.C & -1.195926 & 1.697677 & 3.670609 \\
\hline 59.C & 2.931388 & -1.956168 & 4.228999 \\
\hline 60.C & -0.445276 & 2.547636 & 4.714190 \\
\hline 61.C & -4.426611 & 1.986091 & 5.256611 \\
\hline 62.C & -0.902562 & -0.848476 & 5.418520 \\
\hline 63.C & -1.249091 & -2.344359 & 5.543325 \\
\hline 64.C & -3.444851 & 0.895889 & 5.725388 \\
\hline 65.C & -4.207761 & -0.163111 & 6.548728 \\
\hline 66.C & -0.523937 & -0.310112 & 6.814802 \\
\hline 67.H & 4.677943 & -0.303432 & -7.431037 \\
\hline 68.H & 1.363559 & 0.406957 & -7.520126 \\
\hline 69.H & -0.313301 & 0.891864 & -7.235122 \\
\hline 70.H & 3.560098 & 0.971714 & -6.913593 \\
\hline 71.H & 0.220539 & -0.744044 & -6.811597 \\
\hline 72.H & 2.747602 & -1.386079 & -6.432348 \\
\hline 73.H & 5.071158 & -2.308875 & -6.091360 \\
\hline 74.H & 2.095586 & 2.509247 & -6.228579 \\
\hline 75.H & 5.018922 & 0.623577 & -5.965912 \\
\hline 76.H & 0.395325 & 2.904673 & -5.957728 \\
\hline 77.H & 1.131976 & -3.000556 & -5.445276 \\
\hline 78.H & -0.300101 & -1.964844 & -5.271745 \\
\hline 79.H & 3.912601 & -2.879487 & -4.881183 \\
\hline 80.H & -3.820010 & 1.632394 & -4.789912 \\
\hline 81.H & 5.092858 & -1.628477 & -4.456102 \\
\hline 82.H & -2.053468 & 1.707384 & -4.846472 \\
\hline 83.H & -0.000721 & 0.787516 & -4.781870 \\
\hline
\end{tabular}




$\begin{array}{crrr}84 . \mathrm{H} & 1.504899 & 2.795694 & -4.579141 \\ 85 . \mathrm{H} & 3.870598 & 1.874127 & -4.550937 \\ 86 . \mathrm{H} & -5.838745 & 2.640714 & -4.276069 \\ 87 . \mathrm{H} & -0.091905 & -3.371900 & -4.221935 \\ 88 . \mathrm{H} & -2.971483 & 3.051401 & -4.144662 \\ 89 . \mathrm{H} & -7.499329 & 2.446400 & -3.694966 \\ 90 . \mathrm{H} & -5.706722 & 0.037048 & -3.942361 \\ 91 . \mathrm{H} & -2.620600 & -4.179621 & -4.115570 \\ 92 . \mathrm{H} & -7.328541 & 0.021757 & -3.237078 \\ 93 . \mathrm{H} & 5.450013 & 0.301325 & -3.402579 \\ 94 . \mathrm{H} & -1.848377 & -0.504865 & -3.672447 \\ 95 . \mathrm{H} & -0.615894 & -5.413769 & -3.494301 \\ 96 . \mathrm{H} & 6.010481 & 1.988937 & -3.294623 \\ 97 . \mathrm{H} & -3.603193 & -0.693778 & -3.517774 \\ 98 . \mathrm{H} & 2.739347 & -3.220729 & -3.297356 \\ 99 . \mathrm{H} & -3.676554 & -5.142387 & -3.071874 \\ 100 . \mathrm{H} & -6.429740 & 3.640196 & -2.937443 \\ 101 . \mathrm{H} & 3.588983 & 2.695396 & -3.023960 \\ 102 . \mathrm{H} & 0.430149 & -1.205958 & -3.038337 \\ 103 . \mathrm{H} & -3.657517 & -3.371411 & -2.929860 \\ 104 . \mathrm{H} & 1.876972 & 4.446790 & -2.870166 \\ 105 . \mathrm{H} & 0.138809 & 4.115571 & -2.872410 \\ 106 . \mathrm{H} & -5.963510 & -0.672246 & -2.339688 \\ 107 . \mathrm{H} & -4.232096 & 4.123375 & -2.434311 \\ 108 . \mathrm{H} & -1.386420 & -6.488767 & -2.322413 \\ 109 . \mathrm{H} & -1.912223 & 1.604456 & -2.365006 \\ 110 . \mathrm{H} & 1.330799 & -3.328100 & -2.229757 \\ 111 . \mathrm{H} & 3.301009 & 6.403884 & -2.109013 \\ 112 . \mathrm{H} & 1.262482 & 2.885833 & -2.280843 \\ 113 . \mathrm{H} & 4.295935 & 4.943174 & -2.157142 \\ 114 . \mathrm{H} & -1.307586 & -3.410603 & -2.130050 \\ 115 . \mathrm{H} & 2.549859 & -2.086238 & -1.952312 \\ 131 . \mathrm{H} & -4.409092 & -7.701382 & -0.371608 \\ 132 . \mathrm{H} & -5.043999 & 5.484800 & -0.572702 \\ 133 . \mathrm{H} & 6.584823 & 2.445076 & -0.508315 \\ 117 . \mathrm{H} & -2.564774 & -0.805651 & -2.090689 \\ 118 . \mathrm{H} & 5.025901 & 6.505529 & -1.730519 \\ 119 . \mathrm{H} & -3.491775 & -6.916203 & -1.668145 \\ 120 . \mathrm{H} & -0.006121 & -5.486505 & -1.834320 \\ 121 . \mathrm{H} & 6.857747 & -0.172165 & -1.677108 \\ 122 . \mathrm{H} & 4.691968 & -1.471045 & -1.841536 \\ 123 . \mathrm{H} & -0.436094 & 6.119255 & -1.578958 \\ 124 . \mathrm{H} & -1.885769 & 3.834537 & -1.703028 \\ 125 . \mathrm{H} & 1.251438 & 6.641387 & -1.560010 \\ 126 . \mathrm{H} & 5.336788 & 3.263633 & -1.477588 \\ 128 . \mathrm{H} & -4.708138 & -1.655980 & -1.039494 \\ & -6.142382 & 4.102512 & -0.735795 \\ 12.911019 & -3.394443 & -1.039750 \\ 130 & -5898 & & \end{array}$




\begin{tabular}{|c|c|c|c|}
\hline 134.H & 5.730844 & -2.068724 & -0.562219 \\
\hline 135.H & -4.748851 & -5.250806 & -0.341527 \\
\hline $136 . \mathrm{H}$ & -2.657542 & -7.494869 & -0.214333 \\
\hline 137.H & 4.924621 & -4.132785 & -0.558783 \\
\hline 138.H & 0.126107 & 4.023477 & -0.378864 \\
\hline 139.H & 0.343794 & 6.531978 & -0.041917 \\
\hline 140.H & 6.407042 & 0.280354 & -0.017383 \\
\hline 141.H & -2.389759 & 3.707626 & -0.010464 \\
\hline 142.H & -6.407280 & -0.280446 & 0.017813 \\
\hline 143.H & 2.657768 & 7.494872 & 0.214387 \\
\hline 144.H & -0.343395 & -6.532227 & 0.041871 \\
\hline 145.H & 4.409346 & 7.701125 & 0.371756 \\
\hline 146.H & 2.389004 & -3.707055 & 0.011129 \\
\hline 147.H & 4.748973 & 5.250710 & 0.341231 \\
\hline 148.H & -4.925182 & 4.132812 & 0.558639 \\
\hline 149.H & -6.584584 & -2.445115 & 0.508709 \\
\hline 150.H & 5.043710 & -5.484746 & 0.572557 \\
\hline 151.H & -5.731334 & 2.068668 & 0.562092 \\
\hline 152.H & -0.126019 & -4.023769 & 0.378964 \\
\hline 153.H & 6.142085 & -4.102436 & 0.735402 \\
\hline 154.H & 4.911035 & 3.394987 & 1.039742 \\
\hline 155.H & 2.588179 & -5.242343 & 0.881583 \\
\hline 156.H & 4.708579 & 1.656477 & 1.040299 \\
\hline 157.H & -5.026156 & -6.505290 & 1.730424 \\
\hline 158.H & -1.251135 & -6.641858 & 1.559894 \\
\hline 159.H & -5.336430 & -3.263509 & 1.477975 \\
\hline 160.H & 3.491866 & 6.915932 & 1.668165 \\
\hline 161.H & -6.857807 & 0.172146 & 1.677569 \\
\hline 162.H & 6.674413 & -1.625357 & 1.494730 \\
\hline 163.H & 0.436318 & -6.119455 & 1.578981 \\
\hline 164.H & 1.885765 & -3.835078 & 1.703833 \\
\hline 165.H & -4.692400 & 1.471524 & 1.841626 \\
\hline 166.H & -3.301250 & -6.403976 & 2.108966 \\
\hline 167.H & 0.006065 & 5.486490 & 1.833858 \\
\hline 168.H & -4.295861 & -4.943058 & 2.156954 \\
\hline 169.H & 1.386296 & 6.488869 & 2.321945 \\
\hline 170.H & 1.307517 & 3.410665 & 2.130150 \\
\hline 171.H & -2.549703 & 2.085608 & 1.951568 \\
\hline 172.H & 2.563915 & 0.805388 & 2.090878 \\
\hline 173.H & 4.232112 & -4.123416 & 2.434290 \\
\hline 174.H & -1.331658 & 3.328300 & 2.229497 \\
\hline 175.H & -1.262448 & -2.886236 & 2.280841 \\
\hline 176.H & 5.963529 & 0.672096 & 2.339604 \\
\hline 177.H & 1.912117 & -1.604706 & 2.365673 \\
\hline 178.H & 6.429742 & -3.640329 & 2.937181 \\
\hline 179.H & -1.877024 & -4.447187 & 2.870129 \\
\hline 180.H & 3.657264 & 3.371496 & 2.930255 \\
\hline 181.H & -0.138842 & -4.116023 & 2.872470 \\
\hline 182.H & 3.676503 & 5.142493 & 3.071911 \\
\hline 183.H & -3.588920 & -2.695378 & 3.023959 \\
\hline
\end{tabular}




\begin{tabular}{|c|c|c|c|}
\hline 184.H & 7.328725 & -0.021929 & 3.236741 \\
\hline $185 . \mathrm{H}$ & -6.010301 & -1.988740 & 3.295054 \\
\hline 186.H & -0.430054 & 1.206354 & 3.038259 \\
\hline 187.H & 0.615632 & 5.414108 & 3.493929 \\
\hline 188.H & -5.449714 & -0.301151 & 3.402919 \\
\hline 189.H & -2.740463 & 3.219889 & 3.296633 \\
\hline 190.H & 7.499317 & -2.446578 & 3.694784 \\
\hline 191.H & 3.603347 & 0.693931 & 3.517301 \\
\hline 192.H & 1.848672 & 0.504812 & 3.673198 \\
\hline 193.H & 5.707037 & -0.037156 & 3.942350 \\
\hline 194.H & 2.620321 & 4.180070 & 4.115714 \\
\hline 195.H & 5.838745 & -2.640977 & 4.275906 \\
\hline 196.H & 2.972087 & -3.051274 & 4.145200 \\
\hline 197.H & 0.091022 & 3.372716 & 4.221606 \\
\hline 198.H & -3.870121 & -1.874117 & 4.551001 \\
\hline 199.H & -5.092672 & 1.628676 & 4.456212 \\
\hline 200.H & -1.504175 & -2.795584 & 4.578916 \\
\hline 201.H & 3.820660 & -1.632085 & 4.789988 \\
\hline 202.H & 2.054156 & -1.707257 & 4.847118 \\
\hline 203.H & 0.001097 & -0.787099 & 4.781560 \\
\hline 204.H & -3.912364 & 2.879551 & 4.881537 \\
\hline 205.H & 0.299421 & 1.965951 & 5.271783 \\
\hline 206.H & -1.133036 & 3.001214 & 5.444712 \\
\hline 207.H & -5.019100 & -0.623352 & 5.965747 \\
\hline 208.H & -5.070874 & 2.31 & 6.091627 \\
\hline 209.H & -0.394422 & -2.904325 & 5.957379 \\
\hline 210.H & -2.094726 & -2.509261 & 6.228426 \\
\hline 211.H & -2.747372 & 1.385767 & 6.432325 \\
\hline 212.H & -0.220024 & 0.744280 & 6.811307 \\
\hline 213.H & -3.560143 & -0.972231 & 6.912936 \\
\hline 214.H & 0.313553 & -0.891689 & 7.234950 \\
\hline $215 . \mathrm{H}$ & -4.677443 & 0.303107 & 7.431071 \\
\hline 216.H & -1.363259 & -0.406542 & 7.519791 \\
\hline 217.N & 2.829507 & 0.693015 & -3.056216 \\
\hline 218.N & 5.184699 & 1.156701 & -1.497606 \\
\hline 219.N & -4.017655 & 0.933454 & -0.127699 \\
\hline 220.N & 3.175378 & 2.680836 & -0.054067 \\
\hline 221.N & -3.175203 & -2.681111 & 0.054411 \\
\hline 222.N & 4.017415 & -0.933344 & 0.127962 \\
\hline 223.N & -5.184630 & -1.156544 & 1.497929 \\
\hline 224.N & -2.829274 & -0.693036 & 3.056079 \\
\hline 225.P & 0.235912 & -1.009171 & 0.052397 \\
\hline 226.P & -0.235851 & 1.009078 & -0.052410 \\
\hline 227.Si & 2.172949 & -0.226532 & -4.422607 \\
\hline 228.Si & -4.226231 & 1.815143 & -1.650743 \\
\hline 229.Si & -2.472313 & -4.296598 & -0.225447 \\
\hline 230.Si & 2.472391 & 4.296417 & 0.225510 \\
\hline 231.Si & 4.226076 & -1.815132 & 1.650839 \\
\hline 232.Si & -2.172762 & 0.226570 & 4.422472 \\
\hline 233.U & 2.560826 & 0.598946 & -0.775251 \\
\hline
\end{tabular}


234.U $\quad-2.560903 \quad-0.598943 \quad 0.775202$

Energy: $-1259.46693015 \mathrm{eV}$

Table S7. Final Coordinates and Energy for Single Point Energy Calculation on Geometry

Optimized 5 (spin septet)

$\begin{array}{lrrr}\text { 1.C } & 0.509580 & 0.343201 & -6.785376 \\ \text { 2.C } & 4.205820 & 0.142120 & -6.609009 \\ \text { 3.C } & 3.444711 & -0.904313 & -5.768227 \\ \text { 4.C } & 1.306275 & 2.369697 & -5.544837 \\ \text { 5.C } & 4.421086 & -2.002532 & -5.307950 \\ \text { 6.C } & 0.936216 & 0.880945 & -5.403047 \\ \text { 7.C } & 0.449287 & -2.506408 & -4.679803 \\ \text { 8.C } & -2.932676 & 1.934277 & -4.188651 \\ \text { 9.C } & -6.475105 & 2.645424 & -3.377123 \\ \text { 10.C } & 1.229898 & -1.659368 & -3.655935 \\ \text { 11.C } & 3.945077 & 1.706044 & -3.507959 \\ \text { 12.C } & -6.276257 & 0.165610 & -3.020093 \\ \text { 13.C } & -3.037074 & -4.262072 & -3.077137 \\ \text { 14.C } & 5.318485 & 1.285263 & -2.992854 \\ \text { 15.C } & -2.742261 & -0.281804 & -2.997730 \\ \text { 16.C } & -2.869953 & 1.240744 & -2.813829 \\ \text { 17.C } & 2.034874 & -2.592935 & -2.733699 \\ \text { 18.C } & -6.038020 & 1.550744 & -2.384219 \\ \text { 19.C } & -0.920182 & -5.478325 & -2.414825 \\ \text { 20.C } & 1.116632 & 3.976423 & -2.321518 \\ \text { 21.C } & -1.902505 & -4.353160 & -2.037595 \\ \text { 22.C } & 4.164020 & 5.926730 & -1.595851 \\ \text { 23.C } & -4.076768 & 3.717195 & -1.408555 \\ \text { 24.C } & 0.521880 & 6.044943 & -1.038579 \\ \text { 25.C } & 6.081772 & 0.037420 & -0.998527 \\ \text { 26.C } & -2.659526 & 4.125704 & -0.982241 \\ \text { 27.C } & -3.558762 & -7.057855 & -0.576378 \\ \text { 28.C } & 5.594677 & 2.448166 & -0.820224 \\ \text { 29.C } & 5.200028 & -1.201510 & -0.863697 \\ \text { 30.C } & 0.975248 & 4.576054 & -0.915481 \\ \text { 31.C } & -5.109309 & 4.389796 & -0.480417 \\ \text { 32.C } & -4.654283 & -2.600090 & -0.373081 \\ \text { 33.C } & 3.840959 & 5.703004 & -0.104073 \\ \text { 34.C } & -3.842138 & -5.702868 & 0.104064 \\ \text { 35.C } & 5.108898 & -4.388588 & 0.480630 \\ \text { 36.C } & 4.652381 & 2.598930 & 0.373265 \\ \text { 37.C } & 3.557845 & 7.058215 & 0.576336 \\ \text { 38.C } & -5.594643 & -2.447459 & 0.822170 \\ \text { 39.C } & -5.201805 & 1.202586 & 0.861520 \\ \text { 40.C } & -6.082093 & -0.036772 & 0.997343 \\ \text { 41.C } & -0.976841 & -4.575456 & 0.916281\end{array}$




\begin{tabular}{|c|c|c|c|}
\hline 42.C & -0.523133 & -6.046030 & 1.038927 \\
\hline 43. C & 2.658563 & -4.126893 & 0.982147 \\
\hline 44.C & -4.164924 & -5.926617 & 1.596247 \\
\hline 45.C & 4.077562 & -3.717403 & 1.408061 \\
\hline 46.C & 1.902223 & 4.353276 & 2.037465 \\
\hline 47.C & 6.037835 & -1.549447 & 2.382647 \\
\hline 48.C & 0.918699 & 5.478422 & 2.414260 \\
\hline 49.C & -1.118418 & -3.977046 & 2.321374 \\
\hline 50.C & -2.034585 & 2.591180 & 2.731318 \\
\hline 51.C & 2.870726 & -1.240692 & 2.814182 \\
\hline 52.C & -5.318395 & -1.281856 & 2.992825 \\
\hline 53.C & 6.276181 & -0.166725 & 3.017146 \\
\hline 54.C & 3.036684 & 4.263801 & 3.076697 \\
\hline 55.C & 2.741268 & 0.282682 & 2.996531 \\
\hline 56.C & 6.476129 & -2.647666 & 3.379149 \\
\hline 57.C & -3.945722 & -1.705749 & 3.508052 \\
\hline 58.C & -1.229987 & 1.658840 & 3.655053 \\
\hline 59.C & 2.934481 & -1.932571 & 4.189000 \\
\hline 60.C & -0.451624 & 2.507058 & 4.679643 \\
\hline 61.C & -4.419246 & 2.003123 & 5.305604 \\
\hline 62.C & -0.934932 & -0.880600 & 5.403376 \\
\hline 63.C & -1.304899 & -2.369428 & 5.544882 \\
\hline 64.C & -3.444196 & 0.904317 & 5.767316 \\
\hline 65.C & -4.206438 & -0.141061 & 6.608558 \\
\hline 66.C & -0.508324 & -0.343190 & 6.786038 \\
\hline 67.H & 4.650171 & -0.331059 & -7.500695 \\
\hline 68.H & 1.329400 & 0.425909 & -7.514818 \\
\hline 69.H & -0.332047 & 0.934476 & -7.183479 \\
\hline 70.H & 3.563225 & 0.961022 & -6.959400 \\
\hline 71.H & 0.191899 & -0.706866 & -6.769627 \\
\hline 72.H & 2.727718 & -1.388666 & -6.459189 \\
\hline 73.H & 5.049979 & -2.337227 & -6.149781 \\
\hline 74.H & 2.135527 & 2.515308 & -6.253686 \\
\hline 75.H & 5.036225 & 0.589511 & -6.042977 \\
\hline 76.H & 0.450970 & 2.943725 & -5.937288 \\
\hline 77.H & 1.117830 & -2.978127 & -5.417075 \\
\hline 78.H & -0.295137 & -1.918650 & -5.231287 \\
\hline 79.H & 3.902335 & -2.887834 & -4.919727 \\
\hline 80.H & -3.813828 & 1.613355 & -4.765017 \\
\hline 81.H & 5.101502 & -1.646190 & -4.519053 \\
\hline 82.H & -2.046031 & 1.681731 & -4.792738 \\
\hline 83.H & 0.050013 & 0.838036 & -4.740456 \\
\hline 84.H & 1.596901 & 2.819890 & -4.590254 \\
\hline 85.H & 3.999648 & 1.846347 & -4.599189 \\
\hline 86.H & -5.835985 & 2.664382 & -4.273021 \\
\hline 87.H & -0.092616 & -3.318058 & -4.170730 \\
\hline 88.H & -2.970544 & 3.029925 & -4.107089 \\
\hline 89.H & -7.507415 & 2.462871 & -3.723593 \\
\hline 90.H & -5.709118 & 0.056202 & -3.954804 \\
\hline 91.H & -2.624687 & -4.171478 & -4.095413 \\
\hline
\end{tabular}




\begin{tabular}{|c|c|c|c|}
\hline 92.H & -7.340179 & 0.028661 & -3.272412 \\
\hline 93.H & 5.558106 & 0.295436 & -3.404538 \\
\hline 94.H & -1.856700 & -0.528117 & -3.603817 \\
\hline 95.H & -0.599872 & -5.375785 & -3.465288 \\
\hline 96.H & 6.117461 & 1.984669 & -3.305552 \\
\hline 97.H & -3.616239 & -0.710164 & -3.506175 \\
\hline 98.H & 2.755752 & -3.203405 & -3.294339 \\
\hline 99.H & -3.674417 & -5.159030 & -3.069058 \\
\hline 100.H & -6.452634 & 3.651667 & -2.936166 \\
\hline 1.H & 3.695418 & 2.698799 & -3.093050 \\
\hline $2 . \mathrm{H}$ & 0.479680 & -1.152956 & -3.016578 \\
\hline 103.H & -3.683035 & -3.389478 & -2.910864 \\
\hline 4.H & 1.914163 & 4.471415 & -2.893922 \\
\hline $5 . \mathrm{H}$ & 0.183691 & 4.100071 & -2.894594 \\
\hline 106.H & -5.983622 & -0.663725 & -2.360273 \\
\hline 107.H & -4.246368 & 4.119705 & -2.424344 \\
\hline 108.H & -1.369931 & -6.476696 & -2.317143 \\
\hline 109.H & -1.943592 & 1.578333 & -2.312218 \\
\hline 110.H & 1.358763 & -3.285628 & -2.209851 \\
\hline 111.H & 3.335685 & 6.428516 & -2.116048 \\
\hline 112.H & 1.339276 & 2.898281 & -2.303276 \\
\hline 113.H & 4.361253 & 4.988303 & -2.133352 \\
\hline 114.H & -1.340995 & -3.400317 & -2.089518 \\
\hline 115.H & 2.602914 & -2.061931 & -1.952789 \\
\hline $116 . \mathrm{H}$ & -2.625779 & -0.831578 & -2.047128 \\
\hline 117.H & 5.051975 & 6.569991 & -1.711149 \\
\hline 118.H & -3.454956 & -6.963418 & -1.665229 \\
\hline 119.H & -0.011341 & -5.460390 & -1.798276 \\
\hline 120.H & -6.718241 & 1.627135 & -1.513536 \\
\hline 121.H & 6.954764 & -0.142241 & -1.653870 \\
\hline 122.H & 4.804666 & -1.471456 & -1.861283 \\
\hline $123 . \mathrm{H}$ & -0.420456 & 6.104239 & -1.606774 \\
\hline 124.H & -1.898425 & 3.805897 & -1.705913 \\
\hline $125 . \mathrm{H}$ & 1.260276 & 6.649932 & -1.585313 \\
\hline 126.H & 5.422759 & 3.279787 & -1.514364 \\
\hline 127.H & -4.770333 & -1.707086 & -1.018590 \\
\hline 128.H & -6.148210 & 4.127075 & -0.727606 \\
\hline 129.H & -4.985558 & -3.443484 & -0.999672 \\
\hline 130.H & -2.581386 & 5.220424 & -0.881016 \\
\hline $131 . \mathrm{H}$ & -4.386342 & -7.762111 & -0.387070 \\
\hline 132.H & -5.025827 & 5.486853 & -0.543071 \\
\hline 133.H & 6.660216 & 2.469215 & -0.523213 \\
\hline 134.H & 5.832493 & -2.053276 & -0.565105 \\
\hline 135.H & -4.764391 & -5.317244 & -0.365479 \\
\hline 136.H & -2.641575 & -7.528410 & -0.194562 \\
\hline 137.H & 4.935409 & -4.115634 & -0.571509 \\
\hline 138.H & 0.168961 & 4.019469 & -0.403561 \\
\hline 139.H & 0.351097 & 6.529424 & -0.068487 \\
\hline 140.H & 6.461143 & 0.303290 & -0.003469 \\
\hline 141.H & -2.394933 & 3.684982 & -0.009876 \\
\hline
\end{tabular}




\begin{tabular}{|c|c|c|c|}
\hline 142.H & -6.461198 & -0.304078 & 0.002544 \\
\hline 143.H & 2.640578 & 7.528412 & 0.194624 \\
\hline 144.H & -0.353007 & -6.529409 & 0.068603 \\
\hline 145.H & 4.385323 & 7.762418 & 0.386725 \\
\hline $6 . \mathrm{H}$ & 2.392764 & -3.683949 & 0.012001 \\
\hline 7.H & 4.763131 & 5.316828 & 0.365866 \\
\hline 48.H & -4.934885 & 4.117553 & 0.570704 \\
\hline 49. H & -6.660557 & -2.468923 & 0.527076 \\
\hline $0 . \mathrm{H}$ & 5.025027 & -5.486520 & 0.541059 \\
\hline $1 . \mathrm{H}$ & -5.835627 & 2.052864 & 0.559675 \\
\hline $2 . \mathrm{H}$ & -0.170128 & -4.019552 & 0.404476 \\
\hline $53 . \mathrm{H}$ & 6.148105 & -4.127260 & 0.727822 \\
\hline 4.H & 4.982943 & 3.439626 & 1.004078 \\
\hline $5 . \mathrm{H}$ & 2.582769 & -5.220832 & 0.878914 \\
\hline $56 . \mathrm{H}$ & 4.766529 & 1.703613 & 1.016067 \\
\hline 157.H & -5.052454 & -6.570046 & 1.711602 \\
\hline 158.H & -1.261744 & -6.649915 & 1.585289 \\
\hline 159.H & -5.421087 & -3.278000 & 1.516581 \\
\hline 160.H & 3.454254 & 6.963943 & 1.665373 \\
\hline 161.H & -6.955292 & 0.142104 & 1.652702 \\
\hline 162.H & 6.718021 & -1.628454 & 1.513240 \\
\hline 163.H & 0.418719 & -6.104651 & 1.606586 \\
\hline 164.H & 1.898847 & -3.809534 & 1.708160 \\
\hline 165.H & -4.808587 & 1.476063 & 1.858800 \\
\hline $166 . \mathrm{H}$ & -3.336211 & -6.427922 & 2.116326 \\
\hline 167.H & 0.009670 & 5.458663 & 1.798126 \\
\hline 168.H & -4.362266 & -4.988093 & 2.133531 \\
\hline 169.H & 1.367556 & 6.476677 & 2.315198 \\
\hline 170.H & 1.341154 & 3.400202 & 2.090175 \\
\hline 171.H & -2.603023 & 2.059528 & 1.951201 \\
\hline 172.H & 2.622702 & 0.830964 & 2.045878 \\
\hline 173.H & 4.246793 & -4.119424 & 2.423957 \\
\hline 174.H & -1.358087 & 3.282708 & 2.206526 \\
\hline 175.H & -1.340287 & -2.898538 & 2.304087 \\
\hline 176.H & 5.984969 & 0.662459 & 2.358002 \\
\hline 177.H & 1.944196 & -1.579164 & 2.313458 \\
\hline 178.H & 6.451907 & -3.651594 & 2.937445 \\
\hline 179.H & -1.916790 & -4.471565 & 2.894100 \\
\hline $180 . \mathrm{H}$ & 3.683654 & 3.392031 & 2.910833 \\
\hline 181.H & -0.185627 & -4.101405 & 2.895892 \\
\hline 182.H & 3.673219 & 5.161662 & 3.068390 \\
\hline 183.H & -3.698217 & -2.698912 & 3.092833 \\
\hline 184.H & 7.340677 & -0.029171 & 3.272195 \\
\hline 185.H & -6.119275 & -1.978490 & 3.305971 \\
\hline 186.H & -0.478564 & 1.152643 & 3.016931 \\
\hline 187.H & 0.599163 & 5.376425 & 3.464834 \\
\hline 188.H & -5.554782 & -0.290971 & 3.403183 \\
\hline 189.H & -2.755062 & 3.202937 & 3.291087 \\
\hline 190.H & 7.506742 & -2.463495 & 3.723073 \\
\hline 191.H & 3.615529 & 0.712309 & 3.503558 \\
\hline
\end{tabular}




\begin{tabular}{|c|c|c|c|}
\hline 192.H & 1.856546 & 0.527910 & 3.603421 \\
\hline 193.H & 5.708104 & -0.055308 & 3.953576 \\
\hline 194.H & 2.624693 & 4.173148 & 4.095418 \\
\hline 195.H & 5.836600 & -2.663438 & 4.272876 \\
\hline 196.H & 2.974489 & -3.027911 & 4.109004 \\
\hline 197.H & 0.090748 & 3.318789 & 4.171112 \\
\hline 198.H & -4.000608 & -1.846471 & 4.599229 \\
\hline 199.H & -5.098021 & 1.647774 & 4.514820 \\
\hline 200.H & -1.596332 & -2.818986 & 4.590223 \\
\hline 201.H & 3.815956 & -1.609335 & 4.765483 \\
\hline 202.H & 2.047942 & -1.680556 & 4.793411 \\
\hline 203.H & -0.048689 & -0.837465 & 4.741008 \\
\hline 204.H & -3.899079 & 2.888284 & 4.919025 \\
\hline 205.H & 0.291960 & 1.920090 & 5.232951 \\
\hline 206.H & -1.121956 & 2.978804 & 5.415273 \\
\hline 207.H & -5.038016 & -0.587014 & 6.043236 \\
\hline 208.H & -5.049936 & 2.337793 & 6.146092 \\
\hline 209.H & -0.449350 & -2.943659 & 5.936324 \\
\hline 210.H & -2.133633 & -2.515310 & 6.254260 \\
\hline 211.H & -2.727249 & 1.388515 & 6.458313 \\
\hline 212.H & -0.188693 & 0.706216 & 6.770252 \\
\hline 213.H & -3.564811 & -0.960968 & 6.958243 \\
\hline 214.H & 0.332069 & -0.935919 & 7.184616 \\
\hline 215.H & -4.649107 & 0.332812 & 7.500613 \\
\hline 216.H & -1.328555 & -0.424324 & 7.515078 \\
\hline 217.N & 2.932798 & 0.698241 & -3.096113 \\
\hline 218.N & 5.266586 & 1.172010 & -1.511490 \\
\hline 219.N & -4.091938 & 0.943880 & -0.097795 \\
\hline $220 . \mathrm{N}$ & 3.246718 & 2.732052 & -0.094323 \\
\hline 221.N & -3.247968 & -2.731310 & 0.092819 \\
\hline 222.N & 4.091451 & -0.943611 & 0.097735 \\
\hline 223.N & -5.265601 & -1.170268 & 1.511267 \\
\hline 224.N & -2.931896 & -0.699851 & 3.096354 \\
\hline 225.P & 0.161416 & -1.006322 & 0.095458 \\
\hline 226.P & -0.157929 & 1.001175 & -0.089552 \\
\hline 227.Si & 2.217471 & -0.209055 & -4.438456 \\
\hline 228.Si & -4.266818 & 1.811211 & -1.632616 \\
\hline 229.Si & -2.513331 & -4.324579 & -0.206280 \\
\hline 230.Si & 2.512315 & 4.325008 & 0.206279 \\
\hline 231.Si & 4.267116 & -1.811111 & 1.632416 \\
\hline 232.Si & -2.216742 & 0.208386 & 4.438151 \\
\hline 233.U & 2.683742 & 0.632228 & -0.809221 \\
\hline 234.U & -2.683629 & -0.632084 & 0.809211 \\
\hline
\end{tabular}




\section{Optimized 7'}

\begin{tabular}{|c|c|c|c|}
\hline 1.C & -3.682250 & -0.366810 & \\
\hline 2.C & -0.356140 & 0.686020 & \\
\hline 3. C & -4.022580 & -0.234360 & -5.22064 \\
\hline 4.C & -1.887850 & 2.544190 & -5.157110 \\
\hline 5.C & -1.556140 & -2.522650 & \\
\hline 6.C & -4.923900 & -1.392830 & \\
\hline 7.C & -1.267050 & 1.191140 & -4.74120 \\
\hline 8.C & 3.724810 & 1.585620 & -4.50791 \\
\hline 9.C & 1.904920 & 3.285420 & -4.07196 \\
\hline 10.C & 1.732880 & -3.739980 & \\
\hline 1.C & -1.671710 & -1.673230 & \\
\hline 2.C & 2.740230 & 2.147080 & \\
\hline 3. $\mathrm{C}$ & 5.114950 & & \\
\hline 14.C & 2.6 & -1.5 & \\
\hline 15.C & 5.377150 & 670 & \\
\hline 6.C & 4.279720 & -5.92 & \\
\hline 17.C & 2.00 & -2.8 & \\
\hline 18.C & -4.2 & 570 & \\
\hline 9.C & -2.3 & 580 & \\
\hline $0 . \mathrm{C}$ & 5.23 & 3.4 & \\
\hline 1.C & 4.5 & -4. & \\
\hline 2.C & -5.5 & 5840 & \\
\hline 3. $\mathrm{C}$ & -1.5 & 550 & \\
\hline 24.C & 5.2 & 0.3 & \\
\hline 25.C & & & \\
\hline 26.C & 6.0 & & \\
\hline 27.C & 1.8 & -5.1 & \\
\hline 28.C & 2.4 & & \\
\hline 29.C & 0.4 & -4.7 & \\
\hline 30.C & -5.026520 & 8310 & \\
\hline 31.C & -1.0 & 1030 & \\
\hline 32. C & -5.9 & -0.3 & \\
\hline 33.C & -4.60 & 100 & \\
\hline 34.C & 6.03 & 0.1 & \\
\hline 35.C & -1.2 & 4.4 & \\
\hline 36.C & -3.767940 & -4.798630 & 0.0799 \\
\hline 37.C & -5.614350 & 2.061250 & 0.2272 \\
\hline 38.C & & -5.8 & \\
\hline 39.C & 5.61 & -2.19 & \\
\hline 40.C & 4.359430 & -3.003460 & 0.7513 \\
\hline 41.C & 2.740780 & 3.405670 & 0.7613 \\
\hline 42.C & -2.850820 & -4.05 & \\
\hline 43.C & -3.777960 & 5.386520 & \\
\hline 44.C & -4.609280 & 2.157270 & 1.3476 \\
\hline & -2.208910 & -5.036020 & \\
\hline & 5.504380 & -0.462580 & 2.2778 \\
\hline
\end{tabular}




\begin{tabular}{|c|c|c|c|}
\hline 47.C & -4.695930 & 5.558420 & 2.509030 \\
\hline 48.C & -5.355060 & -2.893420 & 2.572340 \\
\hline 49.C & 4.425980 & 0.503570 & 2.750030 \\
\hline $50 . \mathrm{C}$ & -1.616540 & 3.851130 & 2.862390 \\
\hline 51.C & -5.478680 & -4.214100 & 3.365280 \\
\hline 52.C & 1.630330 & -3.031250 & 3.091420 \\
\hline 53.C & -2.481180 & -1.840300 & 3.139470 \\
\hline 54.C & -0.978360 & 5.170280 & 3.314200 \\
\hline 55.C & -5.897190 & -1.727840 & 3.409130 \\
\hline 56.C & -2.611670 & -0.328440 & 3.421410 \\
\hline 57.C & -2.442500 & 3.257350 & 4.013260 \\
\hline 58.C & 1.167220 & -1.923070 & 4.064170 \\
\hline 59.C & 1.760770 & 2.441880 & 4.281450 \\
\hline 60.C & -2.572140 & -2.582420 & 4.477850 \\
\hline 61.C & 1.092560 & 1.089720 & 4.480470 \\
\hline 62.C & 4.352130 & -1.767720 & 5.553950 \\
\hline 63.C & 0.892900 & -2.579570 & 5.428880 \\
\hline 64.C & 3.370500 & -0.595880 & 5.675700 \\
\hline 65.C & 0.376040 & 1.030280 & 5.852660 \\
\hline 66.C & 4.066200 & 0.594890 & 6.321160 \\
\hline 67.H & -4.512270 & -0.434890 & -7.249620 \\
\hline $68 . \mathrm{H}$ & -3.179650 & 0.422150 & -7.024830 \\
\hline 69.H & -3.140720 & -1.170830 & -6.876680 \\
\hline 70.H & -0.910170 & 0.412710 & -6.649100 \\
\hline 71.H & 0.248230 & 1.405410 & -6.166170 \\
\hline 72.H & -2.590400 & 2.390770 & -5.822950 \\
\hline 73.H & -1.113700 & -1.995650 & -5.712420 \\
\hline 74.H & -5.738850 & -1.376650 & -5.362630 \\
\hline 75.H & -1.192740 & 3.118030 & -5.542000 \\
\hline 76.H & 0.169390 & -0.079680 & -5.575020 \\
\hline 77.H & -2.451770 & -2.779360 & -5.317750 \\
\hline 78.H & 3.240430 & 1.354350 & -5.327780 \\
\hline 79.H & -4.578630 & 0.592110 & -5.133300 \\
\hline $80 . \mathrm{H}$ & -4.451600 & -2.239270 & -4.962450 \\
\hline 81.H & 1.401680 & 2.950260 & -4.843330 \\
\hline 82.H & -1.029920 & -3.328800 & -4.831020 \\
\hline 83.H & 4.406090 & 2.260220 & -4.710230 \\
\hline 84.H & 1.126100 & -3.290810 & -4.645160 \\
\hline 85.H & 2.577190 & -3.943030 & -4.474560 \\
\hline 86.H & 2.498670 & 4.010130 & -4.359240 \\
\hline 87.H & -2.273850 & 2.982380 & -4.369880 \\
\hline 88.H & 4.689970 & 4.365560 & -3.984510 \\
\hline 89.H & 4.156620 & 0.783600 & -4.146260 \\
\hline 90.H & 4.905340 & -3.548890 & -3.851970 \\
\hline 91.H & -5.161560 & -1.308490 & -3.871530 \\
\hline 92.H & -0.647080 & 1.405650 & -3.986460 \\
\hline 93.H & 3.803960 & -5.783050 & -3.608700 \\
\hline 94.H & 3.439900 & -1.743240 & -3.873450 \\
\hline $95 . \mathrm{H}$ & 2.002190 & -1.040390 & -3.896850 \\
\hline 96.H & 1.321790 & -4.572750 & -3.707450 \\
\hline
\end{tabular}




$\begin{array}{crrr} & & & \\ 97 . \mathrm{H} & 6.009020 & 5.012400 & -3.349240 \\ 98 . \mathrm{H} & -4.348390 & 1.901970 & -3.597700 \\ 99 . \mathrm{H} & 6.235030 & -4.126110 & -3.173730 \\ 100 . \mathrm{H} & 1.280430 & 3.622980 & -3.396260 \\ 101 . \mathrm{H} & 5.142210 & -6.356020 & -2.944110 \\ 102 . \mathrm{H} & -0.739780 & -1.467270 & -3.419520 \\ 103 . \mathrm{H} & 2.093720 & 1.407320 & -3.285210 \\ 104 . \mathrm{H} & 4.571620 & 5.367730 & -2.742230 \\ 105 . \mathrm{H} & -3.223820 & -2.787960 & -2.893870 \\ 106 . \mathrm{H} & 5.829340 & 2.788020 & -2.619250 \\ 107 . \mathrm{H} & 5.535510 & -2.815980 & -2.576870 \\ 108 . \mathrm{H} & -5.781140 & 0.306620 & -2.511590 \\ 109 . \mathrm{H} & 2.908770 & -0.963900 & -2.580330 \\ 110 . \mathrm{H} & 3.742810 & -6.504680 & -2.181610 \\ 111 . \mathrm{H} & -1.781710 & -3.371520 & -2.519960 \\ 112 . \mathrm{H} & -6.237420 & 1.811420 & -2.181400 \\ 113 . \mathrm{H} & 1.112220 & -2.568760 & -2.449030 \\ 114 . \mathrm{H} & -3.935600 & 2.485470 & -2.161390 \\ 115 . \mathrm{H} & -0.822690 & 4.162520 & -2.138770 \\ 116 . \mathrm{H} & 2.152330 & 5.182670 & -2.024740 \\ 117 . \mathrm{H} & 5.736380 & 0.893920 & -1.932960 \\ 118 . \mathrm{H} & -2.394240 & 4.129200 & -1.838920 \\ 119 . \mathrm{H} & 5.050360 & -0.538270 & -1.716490 \\ 120 . \mathrm{H} & 1.764810 & -5.802320 & -1.591970 \\ 121 . \mathrm{H} & -2.330430 & -2.061620 & -1.782710 \\ 122 . \mathrm{H} & 6.844820 & 4.314300 & -1.157870 \\ 123 . \mathrm{H} & 5.072620 & -4.758210 & -1.259370 \\ 124 . \mathrm{H} & -1.485290 & 2.859750 & -1.487070 \\ 125 . \mathrm{H} & -4.748130 & -1.588420 & -1.272780 \\ 126 . \mathrm{H} & -0.038930 & -4.437510 & -1.302800 \\ 127 . \mathrm{H} & 3.305790 & 5.444060 & -0.946340 \\ 128 . \mathrm{H} & -0.398200 & 6.109550 & -0.939910 \\ 129 . \mathrm{H} & -4.061170 & 5.367730 & -0.771460 \\ 130 . \mathrm{H} & -6.793430 & -0.485720 & -0.700640 \\ 134 . \mathrm{H} & -0.058210 & -5.533470 & -0.136760 \\ 145 . \mathrm{H} & -5.483870 & -2.359520 & -0.073820 \\ 146 . \mathrm{H} & 0.432260 & -4.042230 & 0.174610 \\ 132 . \mathrm{H} & -4.522530 & 3.215170 & -0.913940 \\ 133 . \mathrm{H} & 5.458950 & 4.518130 & -0.384080 \\ 134 . \mathrm{H} & -1.889690 & 6.362510 & -0.418260 \\ 135 . \mathrm{H} & 1.768670 & 5.508870 & -0.505580 \\ 136 . \mathrm{H} & 6.196910 & 3.098730 & -0.342730 \\ 137 . \mathrm{H} & -3.247540 & -5.476010 & -0.400170 \\ 138 . \mathrm{H} & 5.926710 & -2.278040 & -0.385990 \\ 139 . \mathrm{H} & 6.916170 & -0.237970 & -0.258660 \\ 140 . \mathrm{H} & -5.586500 & 2.883010 & -0.324230 \\ 141 . \mathrm{H} & -4.870000 & 6.546210 & -0.052190 \\ & -5.404070 & 5.040360 & 0.034930 \\ 1420 & & & \end{array}$




\begin{tabular}{|c|c|c|c|}
\hline 147.H & -0.385330 & 4.025450 & 0.184830 \\
\hline 148.H & 6.169830 & 1.012770 & 0.416190 \\
\hline 149.H & 1.874430 & -6.669270 & 0.564220 \\
\hline 150.H & -4.498430 & -5.232790 & 0.567770 \\
\hline 151.H & -6.527360 & 1.965620 & 0.598600 \\
\hline 152.H & 4.553120 & -3.962340 & 0.599320 \\
\hline 153.H & -0.682850 & 6.259230 & 0.627640 \\
\hline 154.H & -2.093560 & -3.715180 & 0.521870 \\
\hline 155.H & -6.250430 & -0.261860 & 0.793730 \\
\hline 156.H & 3.644420 & 3.731570 & 0.955250 \\
\hline 157.H & 6.329650 & -2.519020 & 1.150240 \\
\hline 158.H & 2.457550 & -5.289650 & 1.127120 \\
\hline 159.H & 2.674110 & 2.460180 & 1.010040 \\
\hline 160.H & -3.181410 & 6.188370 & 1.333910 \\
\hline 161.H & 2.088800 & 3.925690 & 1.276040 \\
\hline 162.H & -1.792410 & -5.765140 & 1.534620 \\
\hline 163.H & -5.985870 & -2.984730 & 1.801730 \\
\hline 164.H & 4.038850 & -2.896720 & 1.681970 \\
\hline 165.H & -4.845830 & 2.908550 & 1.947070 \\
\hline 166.H & -4.618440 & 1.321860 & 1.878760 \\
\hline 167.H & -5.470530 & 4.964730 & 2.419100 \\
\hline 168.H & -5.000690 & 6.488440 & 2.558940 \\
\hline 169.H & 4.461890 & 1.333670 & 2.211770 \\
\hline 170.H & 6.397580 & -0.054380 & 2.403200 \\
\hline 171.H & 1.547520 & -2.711370 & 2.168780 \\
\hline 172.H & -2.893070 & -5.402760 & 2.638520 \\
\hline 173.H & -1.525830 & -4.574030 & 2.569520 \\
\hline 174.H & -5.131570 & -4.953570 & 2.824240 \\
\hline $175 . \mathrm{H}$ & -0.281310 & 5.431110 & 2.676530 \\
\hline 176.H & 5.466040 & -1.294410 & 2.813000 \\
\hline 177.H & -2.455740 & 0.173030 & 2.594060 \\
\hline 178.H & -5.830300 & -0.896640 & 2.893780 \\
\hline 179.H & -0.854720 & 3.218200 & 2.724830 \\
\hline 180.H & -1.552270 & -1.985210 & 2.798620 \\
\hline 181.H & -4.203580 & 5.331890 & 3.325770 \\
\hline 182.H & -1.664600 & 5.869050 & 3.352040 \\
\hline 183.H & -6.420760 & -4.380010 & 3.578960 \\
\hline 184.H & 1.071640 & -3.826950 & 3.212890 \\
\hline 185.H & 2.565620 & -3.257580 & 3.275910 \\
\hline 186.H & -6.835280 & -1.896710 & 3.636050 \\
\hline 187.H & 2.103640 & 2.505140 & 3.365590 \\
\hline 188.H & 4.583020 & 0.742500 & 3.697720 \\
\hline 189.H & -2.867240 & 2.425690 & 3.715350 \\
\hline 190.H & -3.512390 & -0.134920 & 3.755910 \\
\hline 191.H & 0.277330 & -1.617720 & 3.725110 \\
\hline 192.H & 0.369600 & 1.037420 & 3.791490 \\
\hline 193.H & -4.962530 & -4.146220 & 4.195400 \\
\hline 194.H & -5.372020 & -1.644390 & 4.232500 \\
\hline 195.H & -0.581480 & 5.053810 & 4.202660 \\
\hline 196.H & -3.133150 & 3.897780 & 4.284220 \\
\hline
\end{tabular}




$\begin{array}{lrrc}\text { 197.H } & -1.949630 & -0.063370 & 4.093750 \\ 198 . \mathrm{H} & -2.508270 & -3.547670 & 4.321230 \\ 199 . \mathrm{H} & 1.106340 & 3.155310 & 4.435090 \\ 200 . \mathrm{H} & -1.854390 & 3.069200 & 4.774310 \\ 201 . \mathrm{H} & 5.088920 & -1.516200 & 4.958880 \\ 202 . \mathrm{H} & -3.428750 & -2.377230 & 4.907420 \\ 203 . \mathrm{H} & 2.501930 & 2.536590 & 4.915500 \\ 204 . \mathrm{H} & -1.837850 & -2.296290 & 5.060400 \\ 205 . \mathrm{H} & 3.886220 & -2.547180 & 5.185140 \\ 206 . \mathrm{H} & 0.256010 & -3.315720 & 5.315070 \\ 207 . \mathrm{H} & 4.836950 & 0.857890 & 5.775920 \\ 208 . \mathrm{H} & 1.731240 & -2.927410 & 5.798270 \\ 209 . \mathrm{H} & -0.175590 & 0.221720 & 5.898520 \\ 210 . \mathrm{H} & -0.193570 & 1.820680 & 5.958060 \\ 211 . \mathrm{H} & 0.516900 & -1.914160 & 6.041810 \\ 212 . \mathrm{H} & 4.708510 & -1.989070 & 6.439670 \\ 213 . \mathrm{H} & 3.438980 & 1.345420 & 6.383660 \\ 214 . \mathrm{H} & 2.727390 & -0.900880 & 6.378210 \\ 215 . \mathrm{H} & 1.043940 & 1.011150 & 6.569430 \\ 216 . \mathrm{H} & 4.370450 & 0.347940 & 7.219300 \\ 217 . \mathrm{N} & -3.157110 & 0.621010 & -2.523360 \\ 218 . \mathrm{N} & 3.939250 & 1.001330 & -0.986450 \\ 219 . \mathrm{N} & -5.282570 & 0.894700 & -0.594020 \\ 220 . \mathrm{N} & 3.317450 & -2.552720 & -0.187240 \\ 221 . \mathrm{N} & -3.830530 & -1.318390 & 0.536830 \\ 222 . \mathrm{N} & 5.295730 & -0.775690 & 0.855310 \\ 223 . \mathrm{N} & -3.244650 & 2.377820 & 0.771810 \\ 224 . \mathrm{N} & 3.095960 & -0.125200 & 2.608360 \\ 225 . \mathrm{P} & 0.229980 & 0.293140 & -1.216670 \\ 226 . \mathrm{P} & -0.197450 & -1.157540 & 0.373830 \\ 227 . \mathrm{P} & 0.078710 & 0.952480 & 0.869200 \\ 228 . \mathrm{Si} & -2.541880 & -0.008580 & -4.016120 \\ 229 . \mathrm{Si} & 3.614970 & 2.504030 & -1.810590 \\ 230 . \mathrm{Si} & 2.931550 & -3.696850 & -1.445920 \\ 231 . \mathrm{Si} & -2.544830 & 3.902570 & 1.198900 \\ 232 . \mathrm{Si} & -3.655810 & -2.504210 & 1.796220 \\ 233 . \mathrm{Si} & 2.263190 & -0.367140 & 4.118850 \\ 234 . \mathrm{U} & -2.570880 & 0.428730 & -0.301690 \\ 235 . \mathrm{U} & 2.630100 & -0.411400 & 0.359440 \\ \mathrm{Energy:} & -1225.56508449 \mathrm{eV} & \\ & & & \end{array}$




\section{References}

1. King, D. M.; Tuna, F.; McInnes, E. J. L.; McMaster, J.; Lewis, W.; Blake, A. J.; Liddle, S. T. Science 2012, 337, 717-720.

2. Velian, A.; Nava, M.; Temprado, M.; Zhou, Y.; Field, R. W.; Cummins, C. C. J. Am. Chem. Soc. 2014, 136, 13586-13589.

3. Velian, A.; Cummins, C. C. J. Am. Chem. Soc. 2012, 134, 13978-13981.

4. Transue, W. J.; Nava, M.; Terban, M. W.; Yang, J.; Greenberg, M. W.; Wu, G.; Foreman, E. S.; Mustoe, C. L.; Kennepohl, P.; Owen, J. S.; Billinge, S. J. L.; Kulik, H. J.; Cummins. C. C. J. Am. Chem. Soc. 2019, 141, 431-440.

5. Riu, M.-L. Y.; Jones, R. L.; Transue, W. J.; Müller, P.; Cummins, C. C. Sci. Adv. 2020, 6, eaaz3168.

6. Sheldrick, G. M. Acta Cryst. Sect. A 2015, 71, 3-8.

7. CrysAlisPRO version 39.46, Oxford Diffraction /Agilent Technologies UK Ltd, Yarnton, England.

8. Sheldrick, G. M. Acta Cryst. Sect. C 2015, 71, 3-8.

9. Dolomanov, O. V.; Bourhis, L. J.; Gildea, R. J.; Howard, J. A. K.; Puschmann, H. J. Appl. Cryst. 2009, 42, 339-341.

10. Farugia, L. J. J. Appl. Cryst. 2012, 45, 849-854.

11. Persistence of Vision (TM) Raytracer, Persistence of Vision Pty. Ltd., Williamstown, Victoria, Australia.

12. Rookes, T. M.; Gardner, B. M.; Balázs, G.; Gregson, M.; Tuna, F.; Wooles, A. J.; Scheer, M.; Liddle, S. T. Angew. Chem. Int. Ed. 2017, 56, 10495-10500.

13. Gardner, B. M.; Cleaves, P. A.; Kefalidis, C. E.; Fang, J.; Maron, L.; Lewis, W.; Blake, A. J.; Liddle, S. T. Chem. Sci. 2014, 5, 2489-2497.

14. Fonseca Guerra, C.; Snijders, J. G.; Te Velde, G.; Baerends, E. J. Theor. Chem. Acc. 
1998, 99, 391-403.

15. Te Velde, G.; Bickelhaupt, F. M.; Van Gisbergen, S. J.; Fonseca Guerra, A. C.; Baerends, E. J.; Snijders, J. G.; Ziegler, T. J. Comput. Chem. 2001, 22, 931-967.

16. Van Lenthe, E.; Baerends, E. J.; Snijders, J. G. J. Chem. Phys. 1993, 99, 4597-4610.

17. Van Lenthe, E.; Baerends, E. J.; Snijders, J. G. J. Chem. Phys. 1994, 101, 9783-9792.

18. Van Lenthe, E.; Ehlers, A. E.; Baerends, E. J. J. Chem. Phys. 1999, 110, 8943-8953.

19. Vosko, S. H.; Wilk, L.; Nusair, M. Can. J. Phys. 1980, 58, 1200-1211.

20. Becke, A. D. Phys. Rev. A. 1988, 38, 3098.

21. Perdew, J. P. Phys. Rev. B. 1986, 33, 8822.

22. Portmann, S.; Luthi, H. P. MOLEKEL: An interactive molecular graphics tool. Chimia 2000, 54, 766-770. 